\title{
Polygenic Adaptation has Impacted Multiple Anthropometric
}

\section{Traits}

\author{
Jeremy J. Berg ${ }^{1,3}$, Xinjun Zhang ${ }^{2}$, Graham Coop ${ }^{1}$ \\ ${ }^{1}$ Center for Population Biology and Department of Evolution and Ecology, University of California, Davis. \\ ${ }^{2}$ Department of Anthropology, University of California, Davis. \\ ${ }^{3}$ Current address: Department of Biological Science, Columbia University, New York, NY
}

To whom correspondence should be addressed: jeremy.jackson.berg@gmail.com, gmcoop@ucdavis.edu

\begin{abstract}
Our understanding of the genetic basis of human adaptation is biased toward loci of large phenotypic effect. Genome wide association studies (GWAS) now enable the study of genetic adaptation in polygenic phenotypes. We test for polygenic adaptation among 187 world-wide human populations using polygenic scores constructed from GWAS of 34 complex traits. We identify signals of polygenic adaptation for anthropometric traits including height, infant head circumference (IHC), hip circumference and waist-to-hip ratio (WHR). Analysis of ancient DNA samples indicates that a north-south cline of height within Europe and and a west-east cline across Eurasia can be traced to selection for increased height in two late Pleistocene hunter gatherer populations living in western and west-central Eurasia. Our observation that IHC and WHR follow a latitudinal cline in Western Eurasia support the role of natural selection driving Bergmann's Rule in humans, consistent with thermoregulatory adaptation in response to latitudinal temperature variation.
\end{abstract}

\section{Author's Note on Failure to Replicate}

After this preprint was posted, the UK Biobank dataset was released, providing a new and open GWAS resource. When attempting to replicate the height selection results from this preprint using GWAS data from the UK Biobank, we discovered that we could not. In subsequent analyses, we determined that both the GIANT consortium height GWAS data, as well as another dataset that was used for replication, were impacted by stratification issues that created or at a minimum substantially inflated the height selection signals reported here. The results of this second investigation, written together with additional coauthors, have now been published (https://elifesciences.org/articles/39725 
along with another paper by a separate group of authors, showing similar issues https://elifesciences.org/articles/39702).

A preliminary investigation shows that the other non-height based results may suffer from similar issues. We stand by the theory and statistical methods reported in this paper, and the paper can be cited for these results. However, we have shown that the data on which the major empirical results were based are not sound, and so should be treated with caution until replicated.

\section{Main Text}

Decades of research in anthropology have identified anthropometric traits that show evidence of biological adaptation to climatic conditions as humans spread around the world over the past hundred thousand years. ${ }^{1,2,3}$ However, it can be challenging to rule out non-heritable environmental factors, ${ }^{4,5}$ as opposed to genetic variation, as the primary cause of these phenotypic differences. ${ }^{6}$ Even for phenotypes where there is some confidence that some of the phenotypic differences among populations are due in part to genetic differences, it is often hard to rule out genetic drift as an alterative explanation to selection. ${ }^{7,8,9}$ The development of population-genetic methods and genomic data resources during the last few decades has enabled the interrogation of adaptive hypotheses and has produced an expanding list of examples of plausible human adaptations. ${ }^{10,11}$ However, such approaches are often inherently limited to detecting adaptation in genetically simple traits via large allele frequency changes at a small number of loci, whereas many adaptations likely involve highly polygenic traits and so are undetectable by most approaches. ${ }^{12,13}$ Genome-wide association studies (GWAS) have now identified thousands of loci underlying the genetic basis of many complex traits. ${ }^{14,15,16}$ These studies offer an unprecedented opportunity to identify adaptation in recent human evolution by detecting subtle shifts in allele frequencies compounded over many GWAS loci. $^{17,18,19,20,21,22,23}$

We conducted a broad screen for evidence of directional selection on variants that contribute to 34 polygenic traits by studying the distribution of their allele frequencies in a dataset of 187 human populations (2158 individuals across 161 populations from the Human Origins Panel ${ }^{24}$ and 2504 individuals across 26 populations of the 1000 Genomes phase 3 panel $^{25}$ ), making use of prior large-scale GWAS for these traits (see Table $\mathbf{5}$ ]). We divided the genome into 1700 non-overlapping and approximately independent linkage blocks ${ }^{26}$ and choose the SNP with the highest posterior probability of association within the block. ${ }^{27,28}$ For each trait, we calculate a polygenic score for each population as a weighted sum of allele frequencies at each of these 1700 SNPs, with the GWAS effect sizes taken as the weights. Figure $\mathbb{W}$ shows the distribution of these scores for height across our population samples.

These polygenic scores should not be viewed as phenotypic predictions across populations. For example, the Maasai and Biaka pygmy populations have similar polygenic scores despite having dramatic differences in height. ${ }^{29}$ Discrepancies between polygenic scores and actual phenotypes may 
be expected to occur either because of purely environmental influences on phenotype, or due to geneby-gene and gene-by-environment interactions. We also expect that the accuracy of these scores when viewed as predictions should decay with genetic distance from Europe (where the GWAS were carried out), due to changes in the structure of linkage disequilibrium (LD) between causal variants and tag SNPs picked up in GWAS, and because GWAS are biased toward discovering intermediate frequency variants, which will explain more variance in the region they are mapped in than outside of it. These caveats notwithstanding, the distribution of polygenic scores across populations is informative about the history of natural selection on a given phenotype, ${ }^{18}$ and a number of striking patterns are visible in their distribution. For example, there is a strong gradient in polygenic height scores running from east to west across Eurasia (Figure $\mathbb{\text { ⿴囗十) }}$

To explore whether patterns observed in the polygenic scores were caused by natural selection, we tested whether the observed distribution of polygenic scores across populations could plausibly have been generated under a neutral model of genetic drift. To understand this null model, consider that a neutrally evolving allele has the same expected frequency across a set of independently evolving sub-populations. However, due to genetic drift, individual sub-populations will deviate from this expected frequency, with the variance of the sub-population frequencies given by $F_{S T} p(1-p)$, where $p$ is the ancestral allele frequency, and $F_{S T}$ is Wright's "fixation index," 30 which can be measured from genome-wide data. ${ }^{17,31}$ Our polygenic scores sum the contributions of a large number of effectively unlinked loci, which under our null model will experience genetic drift independently. It follows that under a model of genetic drift, the polygenic score of each of a set of independent sub-populations will be normally distributed, with variance of $V_{A} F_{S T}$, where $V_{A}$ is the additive genetic variance of polygenic scores the ancestral population. Our test is based on a generalization of this simple relation in which we account for both variance and covariance among multiple populations that are non-independent due to common descent, migration, and admixture over the history of human evolution. Specifically, we model the joint distribution of polygenic scores as multivariate normal and use a generalized variance statistic $\left(Q_{X}\right)$ to measure the over-dispersion of polygenic scores relative to the neutral prediction, which is taken as evidence in favor of natural selection driving difference among populations in polygenic scores (see Methods and our previous study ${ }^{18}$ for details). Our approach is similar to classic tests of adaptation on phenotypes measured in common gardens, which rely on comparisons of the within and among-population additive genetic variance for phenotypes and neutral markers, i.e. $Q_{S T} / F_{S T}$ comparisons. ${ }^{32,33,34}$ Importantly, the neutral distribution we derive holds independent of whether the loci truly influence the trait in an additive manner (with respect to each other or the environment), and whether the GWAS loci are truly causal or merely imperfect tags. However, population structure in the original GWAS panels can confound signals of polygenic adaptation. ${ }^{18,20}$ Modern methods are generally considered to be effective at controlling for the effects of population structure, ${ }^{35}$ and we proceed assuming that it has been adequately accounted for in the original GWAS panels. 
We applied our test to each of the 34 traits across all populations, as well as within nine restricted regional groupings (Figure $\boldsymbol{\square}$ and Table $\mathbf{S 3}$ ). Using our test across all populations as a general test for the impact of selection anywhere in the dataset, we find 5 signals of selection after controlling for multiple testing $(p<0.05 / 34)$. In each case of significant over-dispersion, the signal represents a small but systematic shift in allele frequency of a few percent across many loci, which would be undetectable by standard population-genetic tests for selection (see Table $\mathbf{5 6}$ ), such that the majority of the variance in polygenic scores is within populations as opposed to among populations (see Table 54). The traits involved include height, infant head circumference (IHC), hip circumference, waist-hip ratio (WHR), and type 2 diabetes (T2D). Although the sixth-strongest signal, waist circumference, failed to meet the multiple-testing correction, we include it in subsequent analyses due to its obvious relationship to WHR. We also found signals of selection on polygenic scores constructed for waist and hip circumference and waist-hip ratio when adjusted for BMI (Table 5.3), but we focus on the unadjusted versions for ease of interpretation. We do not replicate a previously reported signal of selection on BMI within Europe, but also note that the previous study used many more SNPs than we have in constructing polygenic scores, which likely explains the difference. ${ }^{20}$

The predominantly European ascertainment of GWAS loci can lead to apparent deviations from neutrality. Therefore all $\mathrm{p}$ values in Figure $\boldsymbol{\nabla}$ and throughout the paper are derived from comparing test statistics against frequency-matched empirical controls, unless otherwise stated (see Text SI.3)). This empirical matching is an important control. For example, the distribution of polygenic scores for Schizophrenia show a signal of over-dispersion under the naive null hypothesis, but not after controlling for the effects of ascertainment. More generally, the ascertainment and selection against disease phenotypes pose difficulties for the interpretation of tests of differentiation. Thus, although we see a signal of selection for decreased T2D polygenic scores in Europe, the interpretation of this signal likely requires the development of more explicit models of selection on disease traits (section SI.4).

\section{The Geography of Selection on Height}

In addition to the known gradient of increased polygenic height scores in northern Europeans relative to southern Europeans (latitude correlation within Europe $p=6.3 \times 10^{-6}$, see $\mathbf{5 2}$ and Methods for statistical details), ${ }^{17,18,19,20,36}$ we also find evidence that that natural selection has impacted polygenic height scores well outside of modern Europe. Polygenic scores decline sharply from west to east across Eurasia in a way that cannot be predicted by a neutral model (longitude correlation across Eurasia, $p=4.46 \times 10^{-15}$; Figure $\square$ ), and they are overdispersed within each of our four population clusters (north, south/central, east, and west) across Asia, as well among Native Americans (Figure च). Does this broadly Eurasian signal represents multiple independent episodes of selection on the genetic basis of height, or can it be explained by ancient selection on one or just a few populations, 
with modern signals reflecting variation in the extent to which modern populations derive ancestry from these ancient populations? For example, the signal of selection on height in East Asia is driven entirely by the Tu population sample, who have the highest polygenic height score among East Asian samples ( $p=0.4329$ for height in East Asia after the Tu are removed). Does this unusually high polygenic score reflect recent selection, or the fact that the Tu derive a proportion of their ancestry from an $\sim 800$-year-old admixture event involving a population resembling modern Europeans ${ }^{37}$ ?

To test whether the height signal within Asia is due to a selective event shared with Europeans, we predicted the polygenic height scores across Asia given the deviation of European populations from the Asian mean, and each of the Asian sample's genome-wide relationship to the European samples (see Figure \$, and Methods for details). We find that this prediction conditioned on Europeans are sufficient to explain most the divergence between the Tu and the other East Asian populations in our dataset (see sky blue dots in Figure B), and eliminate the signal of selection among East Asian populations ( $p=0.099$ after conditioning). In fact, all signals of differential selection on height across Asia can be eliminated using these conditional predictions ( $p=0.2019$ after conditioning). This suggests that most of the selected divergence in our polygenic height scores across Eurasia can be attributed either to events which are predominantly ancestral to modern Europeans (but which have impacted other regions via admixture), or which lie along an early lineage which has contributed ancestry broadly across Eurasia.

To gain further clarity about the history of selection on height, we examined polygenic height scores in a set of ancient DNA samples from Western Eurasia. ${ }^{19,38,39}$ In Figure $\$$ 円 we plot estimates of the polygenic score through time for ancient and modern samples, and in Figure $\mathbb{B B}$ a heatmap of signed p-values from our test of selection applied to pairs of populations (for more detail see Text 5[.5). The earliest unambiguous signal of selection for increased height is found approximately 15,000 years ago in the Villabruna cluster of hunter-gatherers, who have significantly increased polygenic scores relative to earlier pleistocene hunter-gatherers (e.g. Villabruna vs Ust'-Ishim $p=0.0015$, Villabruna vs Kostenki14 $p=0.0244$, Villabruna vs Vestonice $p=0.003$ ). The Mal'ta sample also appears to have an elevated polygenic score, on par with modern Europeans, but it is not significantly different from the earlier pleistocene hunter-gatherers in pairwise tests. Moving into the Holocene, the western, Scandanavian, and Caucasus hunter-gatherers (WHG, SHG, and CHG respectively) all have signficiantly increased polygenic height scores when compared to any of the early pleistocene hunter-gatherers. While WHG and SHG share a significant amount of ancestry with the Villabruna cluster, CHG do not, having separated approximately 46kya (along with Mal'ta and the Eastern hunter-gatherers: EHG) from the lineage leading to Villabruna/WHG. ${ }^{40,38}$ Many ancient samples have ancestry nested within this split between Villabruna/WHG and CHG, but seemingly do not inherit a signal of selection for increased height (including pleistocene hunter-gatherers Kostenki14 and Vestonice $\left.{ }^{41,38}\right)$. It is therefore unlikely that the signals we observe can be traced to a single selective event common to Villabruna/WHG/SHG and to CHG. Instead, our results are potentially 
consistent with at least two independent episodes of selection for increased height among pleistocene and/or holocene hunter-gatherers: at least one in the west, affecting Villabruna, WHG, and SHG, and one in the east, affecting CHG (and potentially Mal'ta).

The Yamnaya-related steppe samples (STP) also show a signal of selection for increased polygenic height scores (e.g. STP-Ust'-Ishim $p=0.001$, STP-Vestonice $p=0.004) .{ }^{19,42}$ This signal is likely due to the fact that they draw $\sim 45 \%$ of their ancestry from a population related to the $\mathrm{CHG},{ }^{19}$ who they are not significantly different from (STP-CHG $p=0.62)$. In turn, the central European Late Neolithic and Bronze Age samples (CLB, including the Corded Ware and Bell Beaker culture) share the high polygenic height signal, and draw much of their ancestry from the expansion of the Yamnaya Steppe people. ${ }^{43,44}$ In contrast, many of the European and Near East early Neolithic samples show little difference in scores relative to the early pleistocene hunter-gatherers and have significantly lower polygenic height scores than Villabruna/WHG/SHG and CHG samples and the populations with Yamnaya ancestry (e.g. Levant-SHG $p=0.001$, Levant-CHG $p=0.01$, LevantSTP $p=0.014)$. We do not find support for Mathieson and colleagues ${ }^{19}$ suggestion of selection for reduced height in Iberian Neolithic samples relative to Anatolian Neolithic $\left(p=0.90\right.$, see also $\left.{ }^{42}\right)$.

Taken together, our results suggest that much of the variation we observe among modern Eurasian populations for polygenic height scores can be traced to variation in the amount of the WHG and Yamnaya/CHG ancestry they have inherited. For example, modern Europeans can be described approximately as a mixture between WHG, Yamnaya, and early Neolithic farmers from Anatolia, ${ }^{43}$ and the variation in the relative proportion of ancestry derived from these three sources explains a substantial amount of the variation in polygenic height scores (see Figure 5T0). ${ }^{19,42}$ Similarly, Yamanaya/CHG ancestry decays from west to east across both northern and southern Asia, ${ }^{40,44}$ consistent with the cline of decreasing polygenic height scores moving from west to east across the continent.

Finally, we note that we can reject neutrality in pairwise comparisons between modern East Asian populations and certain ancient samples that do not appear to be involved in the signal of selection for increased height in the west (e.g. CHB-EHG $p=0.004$, CHB-Levant $p=0.014$, Mal'ta-CHB $p=0.006)$. As these ancient populations are distantly related to one another, and show no other signals of selection on height, this may indicate that selection drove polygenic height scores down somewhere in the history of East Asians. However, the intepretation of this signal is complicated by the fact that we cannot completely exclude that polygenic height scores were selected up in these ancient populations. Clarifying this signal will likely require investigation via more explict models of human demographic history ${ }^{23}$ as well as the incorporation of height GWAS from East Asia. 


\section{Selection on Body Shape Polygenic Scores}

As four out of the next five strongest signals beyond height also represent anthropometric traits, we focus the remainder of our efforts on these phenotypes. Due to genetic correlations between traits, it is possible that signals of selection on two (or more) distinct phenotypes actually represent only a single episode of selection, where one trait responds indirectly to selection on the correlated trait. Because the genetic correlation with height varies among these phenotypes (hip circumference: $r=0.39$, IHC: $r=0.268$, waist circumference: $r=0.22$, and WHR: $r=-0.08),{ }^{45,46}$ we expect $a$ priori that signals for more tightly correlated phenotypes are more likely due to a correlated response to selection on height, whereas for example the WHR signal is more likely to be independent.

To test whether the new signals we observe represent selective events distinguishable from the height signal, we developed a multi-trait extension to our null model based on the quantitativegenetic multivariate-selection model of Lande and Arnold ${ }^{47}$ (see Methods and Supplementary Text Section 51.6$)$. We condition on the observed polygenic height scores, and test whether the signal of selection on a second trait is still significant after accounting for a genetic correlation with height (a non-significant p-value is consistent with a correlated response to selection on height). Applying this test to our entire panel of populations, we find that conditioning on height ablates much of the signal for hip circumference $\left(p=0.0186\right.$ compared to $p=1.12 \times 10^{-4}$ when not conditioning on height), whereas signals in IHC $\left(p=1.11 \times 10^{-5}\right.$ vs $\left.p=5.37 \times 10^{-8}\right)$ and WHR $\left(p=3.57 \times 10^{-8}\right.$ vs $p=3.38 \times 10^{-7}$ ) are less affected. Restricting to European populations only, height is better able to explain hip circumference $\left(p=0.1152\right.$ vs $\left.p=3.4 \times 10^{-} 3\right)$, waist circumference $(p=0.0104$ vs $\left.p=2.63 \times 10^{-3}\right)$, and IHC $\left(p=5.1 \times 10^{-3}\right.$ vs $\left.p=1.41 \times 10^{-8}\right)$ signals, while the signal of selection on WHR again remains strong even after conditioning on height $\left(p=1.92 \times 10^{-8}\right.$ vs $\left.p=6.03 \times 10^{-10}\right)$. WHR is genetically correlated within populations with hip $(r=0.316)$ and waist circumference $(r=0.729)$, but not with IHC $(r=0.01) \cdot{ }^{45,46}$ Conditioning on WHR is sufficient to explain waist circumference (global $p=0.1523$ vs $p=3 \times 10^{-3}$, Europe $p=0.5178$ vs $p=2.6 \times 10^{-3}$ ), but signals in HIP, IHC, and height are all independent of WHR (see Table 54). Together, these results suggest that we can distinguish the action of natural selection along a minimum of two phenotypic dimensions (i.e. height and WHR, or unmeasured phenotypes closely correlated to them). The signal of selection observed for hip circumference is likely due at least in part to selection on height, and the waist circumference signal is probably due to selection on a combination of height and WHR (or closely correlated phenotypes; we provide additional evidence for this claim in supplement section 5162). Whereas IHC shows some evidence of being influenced by selection on height, a correlated response to height seems not to fully explain this signal.

Signals of divergence for both IHC and WHR polygenic scores are confined mostly to Europe and West Asia. For both traits the null model gives a significantly improved fit to the data when conditioned on Europe to explain West Asia and similar when conditioning on West Asia to explain 
Europe (Table 55). This suggests that, as is the case for Eurasian height scores, a substantial fraction of the divergence in IHC and WHR polygenic scores among modern populations across western Eurasia reflects divergence among ancient populations and subsequent mixture rather than recent selection.

\section{Bergmann's Rule and Thermoregulatory Adaptation}

For both IHC and WHR, the selective signal in Western Eurasia can be captured in large part by strong, positive latitudinal clines $\left(p=3.16 \times 10^{-15}\right.$ for IHC and $p=3.16 \times 10^{-7}$ for WHR; Figure

[). These clines in polygenic scores support independent phenotypic evidence for larger and wider bodies and rounder skulls at high latitudes, ${ }^{48,1,49,2,50,51,3}$ consistent with Bergmann's Rule, ${ }^{52,53}$ and add genetic support for a thermoregulatory hypothesis for morphological adaptation, whereby individuals in colder environments are thought to have adapted to improve heat conservation by decreasing their surface area to volume ratio.

A broad range of selective mechanisms have been proposed to act on height variation. ${ }^{54}$ Because we do not detect any signal of selection on age at menarche, we think it unlikely that the height signal represents a correlated response due to life-history mediated selection on age at reproductive maturity. ${ }^{55}$ It has also been suggested that selection on height may be explained as a thermoregulatory adaptation. ${ }^{54}$ However, because the surface area to volume ratio is approximately independent of height, ${ }^{56,2}$ the effect of height SNPs on this ratio is mediated almost entirely through their effect on circumference (hip and/or waist; see section $\mathbf{S L 8}$ ). Because the signal of selection on height cannot be explained by conditioning on hip and waist circumference, it seems that the thermoregulation hypothesis cannot fully explain the signal of selection on height.

A second eco-geographic rule relevant to height is Allen's rule, ${ }^{57}$ which predicts relatively shorter limbs in colder environments, again consistent with adaptation on the basis of thermoregulation. In support of this, human populations in colder environments are observed to have proportionally shorter legs, compared to those in warmer environments. ${ }^{49,58}$ However, we detect no signal of selection on polygenic scores for the ratio of sitting to standing height (SHR); a measure of leg length relative to total body height. ${ }^{59}$ Indeed, by combining our height SNPs with their effect on SHR, we find a strong signal that both increases in leg length and torso length underlie the selective signal on height from North to South within Europe, and from East to West across Eurasia (see SL.9). This again suggests that thermoregulatory concerns are unlikely to fully explain signals of selection on height.

\section{Discussion}

The study of polygenic adaptation provides new avenues for the study of human evolution, and promises a new synthesis of physical anthropology and human genetics. Here, we undertake a broad 
scan for evidence of polygenic adaptive divergence among modern human populations, with body size and shape phenotypes providing most of our strongest signals. We show for the first time that it is possible to reject a neutral model of evolution at height associated loci in comparissons between populations outside of Europe. Using ancient DNA, we show that patterns seen across modern populations are consistent with two independent episodes of selection for increased height in pleistocene hunter-gatherer populations that lived in western and west-central Eurasia during or shortly after the last glacial maximum, and then distributed ancestry widely across the continent. We also provide evidence for adaptive divergence of IHC and WHR in western Eurasia, independent of selection on height, and show that signals of selection on hip and waist circumference can likely be explained as correlated responses to selection on height and WHR (or some other closely correlated phenotypes).

It is conspicuous that the signals of adaptive divergence that we detect are mostly localized to western Eurasia, even in cases where it seems implausible that observed phenotypic differences could have been generated under neutrality (e.g. Maasai vs Biaka pygmy). However, the fact that we do not detect departures from neutrality in such cases should not necessarily be taken as evidence against selection. We should expect to be better-powered to detect selective events in populations more closely related to Europeans for two reasons. First, changes in the structure of linkage disequilibrium (LD) across populations should lead GWAS variants to tag causal variation best in populations genetically close to the European-ancestry GWAS panels. ${ }^{60}$ Second, gene-byenvironment and gene-by-gene interactions can lead to changes in the additive effects of individual loci among populations, ${ }^{61}$ and therefore in the way that they respond to selection on the phenotype. We expect that these difficulties can be overcome or mitigated in the future through a combination of well-powered GWAS in multiple populations of non-European ancestry, access to a wider array of ancient DNA samples, and improved frameworks for the interpretation of signals of polygenic adaptation. $^{23}$

The existence of latitudinal trends in the polygenic scores for WHR and IHC support the notion that some of the clinal phenotypic variation in body shape typically thought to represent thermoregulatory adaptation can be attributed to genetic variation driven by selection, while the ability of simple models to unify signals across broad geographic regions again suggests that these patterns could have been generated by a limited number of selective events. Evidence for adaptation on the basis of specific environmental pressures is most convincing when multiple populations independently converge on the same phenotype in the face of the same environmental pressure, a pattern for which we currently lack evidence. Therefore, while our evidence is consistent with adaptation to temperature environments, alternative explanations (e.g. adaptation to diet) are plausible. 


\section{Methods}

\subsection{Population Genetics Datasets}

We downloaded the 1000 genomes phase 3 release data from the 1000 genomes Itp portal. ${ }^{25}$ We also used data from the Human Origins fully public panel ${ }^{24}$ which was imputed from the 1000 Genomes phase 3 as reference, using the Michigan imputation server, ${ }^{62}$ and restricting to SNPs with an imputation quality score (in terms of predicted $r^{2}$ ) of 0.8 or greater (pers. comm. Joe Pickrell). The original genotype data can be downloaded from the Reich lab website (https://reich.hms.harvard.edu/datasets). This combined dataset represent samples from 2504 people from 26 populations in the 1000 Genomes dataset and 2158 people across 161 populations from the Human Origins dataset, for a total of 4662 samples from 187 populations (52). For global analyses we include all 187 populations. In regional analyses we exclude populations with a significant recent (i.e. $<500$ years) African/non-African admixture to avoid confounding admixture with signals of recent selection within regions (see $\mathbf{5 2}$ and S] for the regions).

\subsection{Selection of GWAS SNPs}

We took public GWAS results for a set of traits ${ }^{28}$ and combined them with additional anthropometric traits from the GIANT consortium and a subset of Early Growth phenotypes contributed by EGG Consortium. Table $\mathbf{S}]$ gives a full list of the traits included in this study and the relevant references. For each trait we selected a set of SNPs with which to construct our polygenic scores as follows. For each SNP, we calculated an approximate Bayes factor summarizing the evidence for association at that SNP via the method of Wakefield, ${ }^{63}$ following Pickrell et al $(2016)^{28}$ (see their supplementary note section 1.2.1). We then used a published set of 1700 non-overlapping linkage disequilibrium blocks ${ }^{26}$ to divide the genome, after which we selected the single SNP with the strongest approximate Bayes factor in favor of association within each block to carry forward for analyses.

\subsection{Polygenic Scores and Null Model}

Given a set of $L$ SNPs associated with a trait $(L \approx 1700)$, we construct the vector of polygenic scores $(\vec{Z})$ across all $M=187$ populations by taking the sum of allele frequencies across the L sites (the vector $\vec{p}_{\ell}$ at site $\ell$ ), weighting each allele's frequency by its effect on the trait $\left(\alpha_{\ell}\right)$ to give

$$
\vec{Z}=\sum_{\ell}^{L} 2 \alpha_{\ell} \vec{p}_{\ell}
$$

For each trait, we construct a null model for the joint distribution of polygenic scores across populations, assuming

$$
\vec{Z} \sim M V N\left(\mu, V_{A} \mathbf{F}\right)
$$


where $\mu=2 \sum_{\ell} \alpha_{\ell} \bar{p}_{\ell}, V_{A}=4 \sum_{\ell} \alpha_{\ell}^{2} \bar{p}_{\ell}\left(1-\bar{p}_{\ell}\right)$. Here $\bar{p}_{\ell}$ is the mean allele frequency across all population samples (weighting all population samples equally), and $\mathbf{F}$ is the $M \times M$ population-level genetic covariance matrix. ${ }^{18}$ All polygenic scores are plotted in centered standardized form $\frac{\vec{Z}-\mu}{\sqrt{V_{A}}}$.

We use the Mahalanobis distance of $\vec{Z}$ from its distribution under the null

$$
Q_{X}=\frac{\vec{Z}^{T} \mathbf{F}^{-1} \vec{Z}}{V_{A}}
$$

as a natural test statistic to assess the ability of the null model to explain the data (see Berg and Coop $(2014)^{18}$ for an extended discussion). This test statistic should be $X^{2}$ with $M-1$ degrees of freedom under neutrality. However, in practice we are concerned that the ascertainment of GWAS loci may invalidate our null model, so we compare the test statistic to an empirical null (see Section ST.31)

\subsection{Latitudinal and Longitudinal Correlations}

We also test for selection-driven correlations between geographic variables (e.g. latitude) and a subset of our polygenic scores (see Berg and Coop (2014) $)^{18}$ and Section SI.] for more details of the test). We take the standardized geographic variable and polygenic scores, and then rotate these vectors by the inverse Cholesky decomposition of the relatedness matrix $\mathbf{F}$. These rotated vectors are in a reference frame where the populations represent independent contrasts under the neutral model. We take as our test statistic the covariance of these rotated vectors. We calculate the significance of the statistic by comparing to a null distribution generated by calculating null sets of polygenic scores assembled from resampled SNPs with derived frequency matched to the CEU population sample so as to mimic the effects of the GWAS ascertainment.

\subsection{Analysis of Ancient DNA.}

We included a combined dataset of 63 Ancient Eurasian human population samples with date estimates from $45 \mathrm{kya}-2.5 \mathrm{kya},{ }^{19,38,39}$ combining these samples into pre-specified analysis clusters we took a set of 19 populations that had $<10 \%$ of height SNPs missing (see Table $\mathbf{5 7}$ for a list of ancient populations included). We compare these to the modern population samples from 1000 genome consortium data. We then took the subset of 724 of our 1700 height associated GWAS SNPs with low levels of missing data in these 19 ancient populations (6.2\% averaged over populations).

Polygenic height scores were calculated as in Eq. (四), for loci with no counts in an ancient population we set to the frequency in the combined rest of sample. We construct the $95 \%$ credible

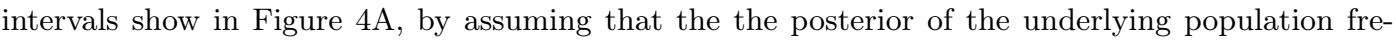
quency is independent across loci and populations and follows a beta distribution, with a uniform prior distribution, which is updated by our binomial sample of ancient counts. Using the variance of the posterior distribution at each locus, we then calculated the variance of the polygenic score $\left(V_{Z}\right)$, 
which follows from Eq. (四). The 95\% credible-interval error bars in Figure 田A were then calculated as $1.96 \sqrt{V_{Z}}$ for each population.

For calculating $Q_{X}($ eqn (B) $)$ ) for pairs of population samples, we restricted the SNP set to the loci that had counts in both samples. Our p-values are calculated assuming that the pairwise $Q_{X}$ statistic has a $\chi^{2}$ distribution, with one degree of freedom. We also constructed a null by flipping the signs of the GWAS effect of the loci at random, and found the $\chi^{2}$ p-values to be well callibrated.

\subsection{Two-Trait Conditional Tests}

Because some of the traits we examine are genetically correlated with one another, we were concerned that signals of selection observed for one trait might reflect a response to selection on another correlated trait. To determine whether genetic correlations might be responsible for some of our signals, we developed a multitrait extension to our neutral model that accounts for genetic covariance among traits. The extension is on the framework of Lande and Arnold. ${ }^{47}$

If $\vec{Z}_{1}$ and $\vec{Z}_{2}$ are vectors of polygenic scores for two different traits constructed according to equation (四), and the matrix $\mathbf{Z}=\left[\vec{Z}_{1}, \vec{Z}_{2}\right]$ contains these vectors as columns, then under neutrality the distribution of $\mathbf{Z}$ is approximately matrix normal

$$
\mathbf{Z} \sim M V N_{M \times 2}(\mu, \mathbf{F}, \mathbf{G})
$$

where the matrix $\mu$ contains the trait-specific means, $\mathbf{F}$ gives the population covariance structure among rows as in the single trait model, and $\mathbf{G}$ is the among trait additive genetic covariance matrix, the "G matrix" of multivariate quantitative genetics, ${ }^{47}$ estimated for a population ancestral to all populations in the sample. The diagonal elements of the $2 \times 2 \mathrm{G}$ matrix are given by the $V_{A}$ parameters from above in the single trait model and the off-diagonal element $\left(C_{A, 12}\right)$ corresponds to the additive genetic covariance between the two traits. Given this null model for the joint distribution of the two traits, we can construct a conditional model for the distribution of polygenic scores for trait 1, given the polygenic score observed for trait 2 , as

$$
\begin{aligned}
\vec{Z}_{1} & \sim \operatorname{MVN}\left(\xi, V_{A C} \mathbf{F}\right) \\
\xi & =\mu_{1}+\frac{C_{A, 12}}{V_{A, 2}}\left(\vec{Z}_{2}-\mu_{2}\right) \\
V_{A C} & =V_{A, 1}-\frac{C_{A, 12}^{2}}{V_{A, 2}} .
\end{aligned}
$$

Given a value of $C_{A, 12}$ we can then use these conditional means and variances in equation (3) to form a conditional $Q_{X}$ statistic and compare it to its null distribution. We take the failure to reject neutrality on the basis of the conditional $Q_{X}$ statistic as consistent with the hypothesis that any response to selection observed for trait 1 is a result of selection on trait 2 . Some of the traits we study have non-linear allometric relationships with each other, but because our polygenic scores are 
linear by construction our tests are robust to this non-linearity (see $\mathbf{5 l . 7}$ ).

We experimented with estimating $C_{A, 12}$ on the basis of SNPs that overlap between the two traits in each genomic block. However, we were concerned about this approach to estimating genetic correlations not being a sufficient joint model for cases in which different SNPs within a block affected the two traits but were in linkage disequilibrium with one another, and therefore do not drift independently. To deal with this issue, we represent the genetic covariance among populations as

$$
C_{A, 12}=\rho \sqrt{V_{A, 1} V_{A, 2}}
$$

where $\rho$ represents the genetic correlation between the two sets of polygenic scores. We pursued a conservative strategy, testing a range of values for $\rho$ along a dense grid from - 1 to 1 to ask whether any assumed genetic correlation between polygenic scores could plausibly allow one trait to be explained as a correlated response to another. As a further conservative measure, we allowed the genetic correlation used to calculate the conditional variance $(\mathrm{Eq}(\mathbb{\pi}))$ to be equal to zero, while allowing the $\rho$ used to compute the conditional mean (Eq (6)) was not. This is a conservative approach, as it fits our conditional prediction to the mean, but allows the variance of the null model to remain as large as the unconditional model. The conditional two-trait p-values we present in the text, and the CI shown in two-trait Figure 5 and in the supplement, use this conservative approach. In practice our values of $\rho$ are consistent with estimates of genetic correlations obtained from the LDscore approach, ${ }^{45,46}$ given that our polygenic scores capture only a fraction of the total genetic variance for each trait.

\subsection{Single Trait Conditional Null Model}

We also developed an extension of the null model for a single trait to test whether two (or more) signals of selection detected in different geographic regions might reflect a single ancestral event that occurred in an ancient population that has contributed ancestry broadly to modern populations.

Assume for example that we have detected a signal of selection among the population samples from region A (e.g. Europe) and among the population samples from (e.g. Asia), and we would like to test whether the signal detected in region $\mathrm{B}$ is due to a selective event that is also responsible for generating a signal of selection in region $\mathrm{A}$. We first reorganize our samples into two blocks for the two regions

$$
\left[\begin{array}{c}
\vec{Z}_{A} \\
\vec{Z}_{B}
\end{array}\right] \sim M V N\left(\left[\begin{array}{l}
\mu_{B} \\
\mu_{B}
\end{array}\right], V_{A}\left[\begin{array}{ll}
\mathbf{F}_{A A} & \mathbf{F}_{A B} \\
\mathbf{F}_{B A} & \mathbf{F}_{B B}
\end{array}\right]\right)
$$

Where $\mu_{B}$ is the mean polygenic score in the set of populations being tested, the $\mathbf{F} \bullet, \bullet$ s refer to the sub-matrices of the relatedness matrix $\mathbf{F}$, and $\mathbf{F}$ itself has been recentered at the mean of the test set (i.e. region B). Then the conditional distribution of polygenic scores in region B given the polygenic 
scores observed in region $\mathrm{A}$ is

$$
\begin{aligned}
\vec{Z}_{B \mid A} \sim M V N\left(\mu_{B \mid A}, V_{A} \mathbf{F}_{B \mid A}\right) \\
\vec{\mu}_{B \mid A}=\mu_{B}+\mathbf{F}_{B A} \mathbf{F}_{A A}^{-1}\left(\vec{Z}_{A}-\mu_{B}\right) \\
\mathbf{F}_{B \mid A}=\mathbf{F}_{B B}-\mathbf{F}_{B A} \mathbf{F}_{A A}^{-1} \mathbf{F}_{A B} .
\end{aligned}
$$

The conditional mean, $\vec{\mu}_{B \mid A}$ reflects the best predictions of population means in region $\mathrm{B}$ given the values observed in region $\mathrm{A}$, whereas the conditional covariance matrix $\mathbf{F}_{B \mid A}$ reflects the scale and form of the variance around this expectation that arises from drift that is independent of drift in the ancestry of populations in region A.

We can then test for over-dispersion of polygenic score in region $B\left(\vec{Z}_{B}\right)$ given the observed polygenic scores in region $A$ by using $\vec{\mu}_{B \mid A}$ and $\mathbf{F}_{B \mid A}$ in (3i) to construct a conditional $Q_{X}$ score. We judge the statistical significance of this conditional $Q_{X}$ score by comparing it to a frequency matched dataset, as with the standard test. We interpret a non-significant conditional $Q_{X}$ score for region $\mathrm{B}$ as evidence that any selective signal of overdispersion in B is well explained by genome-wide allele-sharing with A. We view this as evidence that the selection signal in B overlaps that in A, due to selection in shared ancestral populations and admixture.

In Figure B we plot the observed polygenic scores for Asia against the predicted polygenic scores $\left(\vec{\mu}_{B \mid A}\right)$ for Asia $(B)$, conditional on the Europe population sample polygenic scores (A). The error bars are $95 \%$ CIs for each population sample, obtained from the variances on the diagonal of $V_{A} \mathbf{F}_{B \mid A}$.

\section{Acknowledgements}

We thank the Coop Lab and Doc Edge, Iain Mathieson, Emily Josephs, Joe Pickrell, Molly Przeworski, David Reich, Jeff Ross-Ibarra, Guy Sella, and Tim Weaver for helpful discussions and feedback on earlier drafts. The work was supported in part by an NSF GRFP (to JJB), the UC Davis Anthropology department (XZ), and NIGMS-NIH RO1 grants GM108779 to GC. JJB was also supported in part by R01 grants GM115889 to Guy Sella and GM121372 to Molly Przeworski. 


\section{References}

${ }^{1}$ Roberts, D. F. Body weight, race and climate. American Journal of Physical Anthropology 11, $533-558$ (1953).

${ }^{2}$ Ruff, C. B. Morphological adaptation to climate in modern and fossil hominids. Am. J. Phys. Anthropol. (1994).

${ }^{3}$ Savell, K. R. R., Auerbach, B. M. \& Roseman, C. C. Constraint, natural selection, and the evolution of human body form. Proc. Natl. Acad. Sci. U.S.A. 113, 9492-9497 (2016).

${ }^{4}$ Bogin, B., Smith, P., Orden, A. B., Varela Silva, M. I. \& Loucky, J. Rapid change in height and body proportions of Maya American children. Am. J. Hum. Biol. 14, 753-761 (2002).

${ }^{5}$ Serrat, M. A., King, D. \& Lovejoy, C. O. Temperature regulates limb length in homeotherms by directly modulating cartilage growth. Proc. Natl. Acad. Sci. U.S.A. 105, 19348-19353 (2008).

${ }^{6}$ Pujol, B., Wilson, A., Ross, R. \& Pannell, J. Are $Q_{S T}-F_{S T}$ comparisons for natural populations meaningful? Molecular Ecology 17, 4782-4785 (2008).

${ }^{7}$ Rogers, A. R. \& Harpending, H. C. Population structure and quantitative characters. Genetics 105, 985-1002 (1983).

${ }^{8}$ Relethford, J. H. Craniometric variation among modern human populations. American Journal of Physical Anthropology 95, 53-62 (1994).

${ }^{9}$ Relethford, J. H. Apportionment of global human genetic diversity based on craniometrics and skin color. American Journal of Physical Anthropology 118, 393-398 (2002).

${ }^{10}$ Tishkoff, S. Strength in small numbers. Science (2015).

${ }^{11}$ Fan, S., Hansen, M. E. B., Lo, Y. \& Tishkoff, S. A. Going global by adapting local: A review of recent human adaptation. Science 354, 54-59 (2016).

${ }^{12}$ Pritchard, J. K. \& Di Rienzo, A. Adaptation-not by sweeps alone. Nat Rev Genet (2010).

${ }^{13}$ Pritchard, J. K., Pickrell, J. K. \& Coop, G. The genetics of human adaptation: hard sweeps, soft sweeps, and polygenic adaptation. Current biology (2010).

${ }^{14}$ Visscher, P. M., Brown, M. A. \& McCarthy, M. I. Five years of GWAS discovery. Am. J. Hum. Genet. (2012).

${ }^{15}$ Price, A. L., Spencer, C. C. A. \& Donnelly, P. Progress and promise in understanding the genetic basis of common diseases. Proc. R. Soc. B 282, 20151684-10 (2015). 
${ }^{16}$ Boyle, E. A., Li, Y. I. \& Pritchard, J. K. An expanded view of complex traits: from polygenic to omnigenic. Cell (2017).

${ }^{17}$ Turchin, M. C., Chiang, C. \& Palmer, C. D. Evidence of widespread selection on standing variation in Europe at height-associated SNPs. Nature (2012).

${ }^{18}$ Berg, J. J. \& Coop, G. A population genetic signal of polygenic adaptation. PLoS Genet (2014).

${ }^{19}$ Mathieson, I. et al. Genome-wide patterns of selection in 230 ancient Eurasians. Nat. Gen. 528, 499-503 (2015).

${ }^{20}$ Robinson, M. R., Hemani, G. \& Medina-Gomez, C. Population genetic differentiation of height and body mass index across Europe. Nature (2015).

${ }^{21}$ Hansen, M. E. B. et al. Shorter telomere length in Europeans than in Africans due to polygenetic adaptation. Hum. Mol. Genet. 25, 2324-2330 (2016).

${ }^{22}$ Field, Y. et al. Detection of human adaptation during the past 2000 years. Science 354, 760-764 (2016)

${ }^{23}$ Racimo, F., Berg, J. J. \& Pickrell, J. K. Detecting polygenic adaptation in admixture graphs. bioRxiv 146043 (2017).

${ }^{24}$ Lazaridis, I. et al. Ancient human genomes suggest three ancestral populations for present-day Europeans. Nat. Gen. 513, 409-413 (2014).

${ }^{25} 1000$ Genomes Project Consortium. A global reference for human genetic variation. Nature 526, $68(2015)$

${ }^{26}$ Berisa, T. \& Pickrell, J. K. Approximately independent linkage disequilibrium blocks in human populations. Bioinformatics (2016).

${ }^{27}$ Pickrell, J. K. Joint analysis of functional genomic data and genome-wide association studies of 18 human traits. Am. J. Hum. Genet. (2014).

${ }^{28}$ Pickrell, J. K., Berisa, T., Liu, J. Z., Ségurel, L. \& Tung, J. Y. Detection and interpretation of shared genetic influences on 42 human traits. Nature (2016).

${ }^{29}$ Martin, A. R. et al. Human Demographic History Impacts Genetic Risk Prediction across Diverse Populations. Am. J. Hum. Genet. 100, 635-649 (2017).

${ }^{30}$ Wright, S. The genetical structure of populations. Ann Eugen 15, 323-354 (1951).

${ }^{31}$ Nicholson, G. et al. Assessing population differentiation and isolation from single-nucleotide polymorphism data. J. R. Stat. Soc. 64, 695-715 (2002). 
${ }^{32}$ Prout, T. \& Barker, J. S. F statistics in Drosophila buzzatii: selection, population size and inbreeding. Genetics 134, 369-375 (1993).

${ }^{33}$ Spitze, K. Population structure in Daphnia obtusa: quantitative genetic and allozymic variation. Genetics 135, 367-374 (1993).

${ }^{34}$ Ovaskainen, O., Karhunen, M., Zheng, C., Arias, J. M. C. \& Merila, J. A New Method to Uncover Signatures of Divergent and Stabilizing Selection in Quantitative Traits. Genetics 189, 621-632 (2011).

${ }^{35}$ Bulik-Sullivan, B. K. et al. LD Score regression distinguishes confounding from polygenicity in genome-wide association studies. Nat. Gen. 47, 291-295 (2015).

${ }^{36}$ Zoledziewska, M., Sidore, C., Chiang, C. \& Sanna, S. Height-reducing variants and selection for short stature in Sardinia. Nature (2015).

${ }^{37}$ Hellenthal, G. et al. A genetic atlas of human admixture history. Science 343, 747-751 (2014).

${ }^{38} \mathrm{Fu}, \mathrm{Q}$. et al. The genetic history of Ice Age Europe. Nature (2016).

${ }^{39}$ Lazaridis, I. et al. Genomic insights into the origin of farming in the ancient near east. Nature 536, 419-424 (2016).

${ }^{40}$ Jones, E. R. et al. Upper Palaeolithic genomes reveal deep roots of modern Eurasians. Nature Communications 6, 1-8 (2015).

${ }^{41}$ Seguin-Orlando, A. et al. Paleogenomics. Genomic structure in Europeans dating back at least 36,200 years. Science 346, 1113-1118 (2014).

${ }^{42}$ Martiniano, R. et al. The population genomics of archaeological transition in west iberia. bioRxiv (2017).

${ }^{43}$ Haak, W. et al. Massive migration from the steppe was a source for Indo-European languages in Europe. Nature 522, 207-211 (2015).

${ }^{44}$ Allentoft, M. E. et al. Population genomics of Bronze Age Eurasia. Nature 522, 167-172 (2015).

${ }^{45}$ Bulik-Sullivan, B., Finucane, H. K., Anttila, V. \& Gusev, A. An atlas of genetic correlations across human diseases and traits. Nature (2015).

${ }^{46}$ Zheng, J. et al. LD Hub: a centralized database and web interface to perform LD score regression that maximizes the potential of summary level GWAS data for SNP heritability and genetic correlation analysis. Bioinformatics 33, 272-279 (2017).

${ }^{47}$ Lande, R. \& Arnold, S. J. The measurement of selection on correlated characters. Evolution 37, 1210-1226 (1983). 
${ }^{48}$ SCHREIDER, E. Geographical distribution of the body-weight/body-surface ratio. Nature 165, 286 (1950).

${ }^{49}$ Roberts, D. Climate and human variability (Addison-Wesley, 1973).

${ }^{50}$ Ruff, C. Variation in Human Body Size and Shape. Annu. Rev. Anthropol. 31, 211-232 (2002).

${ }^{51}$ Katz, D. C., Grote, M. N. \& Weaver, T. D. A mixed model for the relationship between climate and human cranial form. Am. J. Phys. Anthropol. 160, 593-603 (2015).

${ }^{52}$ Bergmann, C. Über die Verhältnisse der Wärmeökonomie der Thiere zu ihrer Grösse. Göttinger Studien 3, 595-708 (1847).

${ }^{53}$ Mayr, E. Geographical character gradients and climatic adaptation. Evolution 10, 105-108 (1956). URL http://www.jstor.org/stable/2406103.

${ }^{54}$ Stulp, G. \& Barrett, L. Evolutionary perspectives on human height variation. Biol Rev 91, 206-234 (2014).

${ }^{55}$ Stearns, S. C., Govindaraju, D. R., Ewbank, D. \& Byars, S. G. Constraints on the coevolution of contemporary human males and females. Proceedings of the Royal Society of London B: Biological Sciences 279, 4836-4844 (2012). URL http://rspb.royalsocietypublishing.org/ content/279/1748/4836. http://rspb.royalsocietypublishing.org/content/279/1748/4836. tull.pdt.

${ }^{56}$ Ruff, C. B. Climate and body shape in hominid evolution. Journal of Human Evolution 21, 81-105 (1991).

${ }^{57}$ Allen, J. A. The Influence of Physical Conditions in the Genesis of Species. Radical Review 1, 108-140 (1877).

${ }^{58}$ Katzmarzyk, P. T. \& Leonard, W. R. Climatic influences on human body size and proportions: ecological adaptations and secular trends. Am. J. Phys. Anthropol. 106, 483-503 (1998).

${ }^{59}$ Chan, Y. et al. Genome-wide Analysis of Body Proportion Classifies Height-Associated Variants by Mechanism of Action and Implicates Genes Important for Skeletal Development. Am. J. Hum. Genet. 96, 695-708 (2015).

${ }^{60}$ Palmer, C. \& Pe'er, I. Statistical correction of the winner's curse explains replication variability in quantitative trait genome-wide association studies. bioRxiv (2017).

${ }^{61}$ Brown, B. C. et al. Transethnic genetic-correlation estimates from summary statistics. The American Journal of Human Genetics 99, 76-88 (2016). 
${ }^{62}$ Das, S. et al. Next-generation genotype imputation service and methods. Nature genetics $\mathbf{4 8}$, 1284-1287 (2016).

${ }^{63}$ Wakefield, J. Bayes factors for genome-wide association studies: comparison with P-values. Genet. Epidemiol. 33, 79-86 (2009).

${ }^{64}$ Perry, J. R. B. et al. Parent-of-origin-specific allelic associations among 106 genomic loci for age at menarche. Nature 514, 92-97 (2014).

${ }^{65}$ Lambert, J. C., Ibrahim-Verbaas, C. A., Harold, D. \& Naj, A. C. Meta-analysis of 74,046 individuals identifies 11 new susceptibility loci for Alzheimer's disease. Nature (2013).

${ }^{66}$ van der Valk, R. J. P. et al. A novel common variant in DCST2 is associated with length in early life and height in adulthood. Hum. Mol. Genet. 24, 1155-1168 (2014).

${ }^{67}$ Horikoshi, M., Yaghootkar, H. \& Mook-Kanamori, D. O. New loci associated with birth weight identify genetic links between intrauterine growth and adult height and metabolism. Nature (2013).

${ }^{68}$ Locke, A. E. et al. Genetic studies of body mass index yield new insights for obesity biology. Nature 518, 197-206 (2015).

${ }^{69}$ Schunkert, H., König, I. R., Kathiresan, S. \& Reilly, M. P. Large-scale association analysis identifies 13 new susceptibility loci for coronary artery disease. Nature (2011).

${ }^{70}$ Jostins, L. et al. Host-microbe interactions have shaped the genetic architecture of inflammatory bowel disease. Nature 491, 119-124 (2012).

${ }^{71}$ Manning, A. K., Hivert, M. F., Scott, R. A. \& Grimsby, J. L. A genome-wide approach accounting for body mass index identifies genetic variants influencing fasting glycemic traits and insulin resistance. Nature (2012).

${ }^{72}$ Estrada, K., Styrkarsdottir, U., Evangelou, E. \& Hsu, Y. H. Genome-wide meta-analysis identifies 56 bone mineral density loci and reveals 14 loci associated with risk of fracture. Nature (2012).

${ }^{73}$ van der Harst, P. et al. Seventy-five genetic loci influencing the human red blood cell. Nature 492, 369-375 (2012).

${ }^{74}$ Teslovich, T. M. et al. Biological, clinical and population relevance of 95 loci for blood lipids. Nature 466, 707-713 (2010).

${ }^{75}$ Wood, A. R., Esko, T., Yang, J., Vedantam, S. \& Pers, T. H. Defining the role of common variation in the genomic and biological architecture of adult human height. Nature (2014).

${ }^{76}$ Cousminer, D. L. et al. Genome-wide association and longitudinal analyses reveal genetic loci linking pubertal height growth, pubertal timing and childhood adiposity. Hum. Mol. Genet. 22, $2735-2747$ (2013). 
${ }^{77}$ Shungin, D. et al. New genetic loci link adipose and insulin biology to body fat distribution. Nature 518, 187-196 (2015).

${ }^{78}$ Taal, H. R. et al. Common variants at 12q15 and 12q24 are associated with infant head circumference. Nat Genet 44, 532-538 (2012).

${ }^{79}$ Gieger, C. et al. New gene functions in megakaryopoiesis and platelet formation. Nature 480, 201-208 (2011).

${ }^{80}$ Okada, Y. et al. Genetics of rheumatoid arthritis contributes to biology and drug discovery. Nature 506, 376-381 (2014).

${ }^{81}$ Ripke, S. et al. Biological insights from 108 schizophrenia-associated genetic loci. Nature 511, 421-427 (2014).

${ }^{82}$ Chan, Y., Salem, R. M., Hsu, Y. \& McMahon, G. Genome-wide analysis of body proportion classifies height-associated variants by mechanism of action and implicates genes important for skeletal .... Am. J. Hum. Genet. (2015).

${ }^{83}$ Morris, A. P., Voight, B. F., Teslovich, T. M. \& Ferreira, T. Large-scale association analysis provides insights into the genetic architecture and pathophysiology of type 2 diabetes. Nature (2012).

${ }^{84}$ Pickrell, J. K. \& Pritchard, J. K. Inference of population splits and mixtures from genome-wide allele frequency data. PLoS Genet (2012).

${ }^{85}$ Zhao, L., Lascoux, M., Overall, A. \& Waxman, D. The characteristic trajectory of a fixing allele: A consequence of fictitious selection that arises from conditioning. Genetics (2013).

${ }^{86}$ Kremer, A. \& Le Corre, V. Decoupling of differentiation between traits and their underlying genes in response to divergent selection. Heredity (Edinb) 108, 375-385 (2012).

${ }^{87}$ Le Corre, V. \& Kremer, A. The genetic differentiation at quantitative trait loci under local adaptation. Mol. Ecol. (2012).

${ }^{88}$ Chan, Y., Lim, E. T., Sandholm, N. \& Wang, S. R. An excess of risk-increasing low-frequency variants can be a signal of polygenic inheritance in complex diseases. Am. J. Hum. Genet. (2014).

${ }^{89}$ Mathieson, I. Selection on height in europe. http://mathii.github.io/review/2015/10/21/ selection-on-height-in-europe (2015).

${ }^{90}$ Huxley, J. Problems of Relative Growth (Methuen, London, 1932).

${ }^{91}$ Huxley, J. S. \& Teissier, G. Terminology of relative growth. Nature 137, 780-781 (1936). 
${ }^{92}$ Cheverud, J. M. Relationships among ontogenetic, static, and evolutionary allometry. American Journal of Physical Anthropology 59, 139-149 (1982). URL http://dx.doi.org/10.1002/ajpa. 1330590204.

${ }^{93}$ Lande, R. Quantitative genetic analysis of multivariate evolution, applied to brain: body size allometry. Evolution 402-416 (1979).

${ }^{94}$ Rice, S. H. The evolution of canalization and the breaking of von baer's laws: Modeling the evolution of development with epistasis. Evolution 52, 647-656 (1998). URL http://www.jstor. org/stable/2411260.

${ }^{95}$ Nieuwboer, H. A., Pool, R., Dolan, C. V., Boomsma, D. I. \& Nivard, M. G. GWIS: Genome-Wide Inferred Statistics for Functions of Multiple Phenotypes. Am. J. Hum. Genet. 99, 917-927 (2016).

URL http://www.sciencedirect.com/science/article/pii/S0002929716303214. 

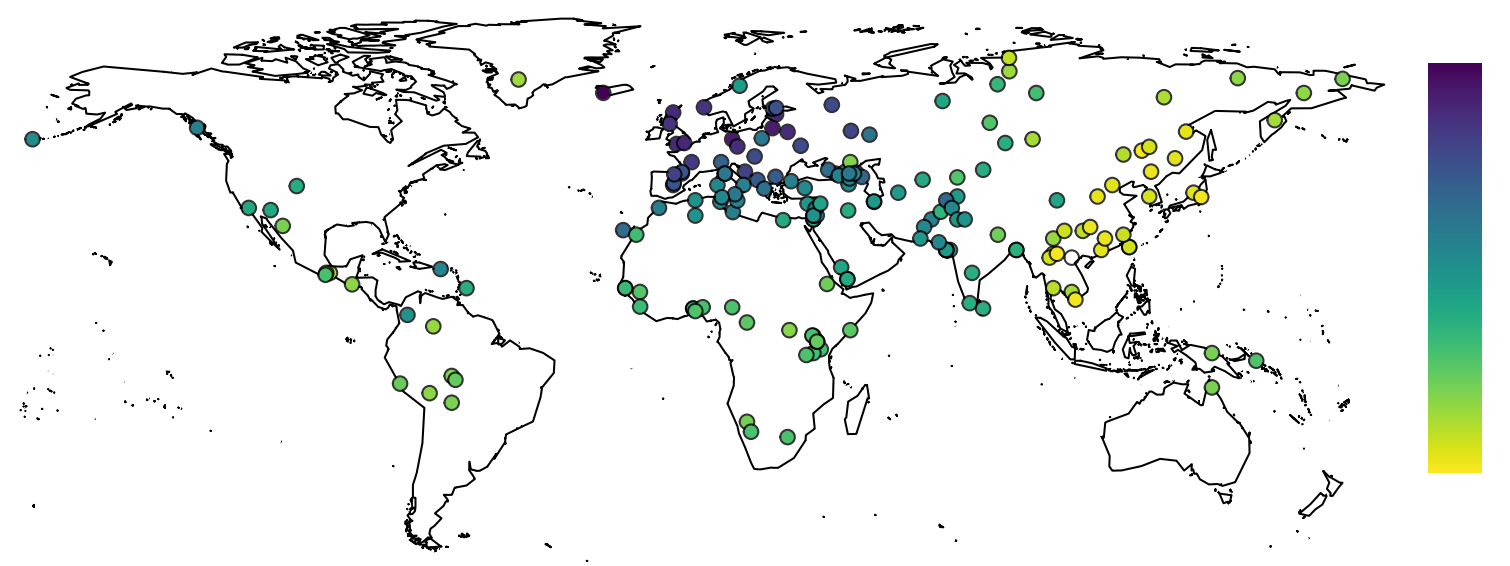

Figure 1: Polygenic Height Scores for 187 population samples (combined Human origin panel and 1000 genomes datasets), plotted on geographic coordinates. Blue corresponds to populations with the "tallest" polygenic height scores, and yellow the "shortest". 


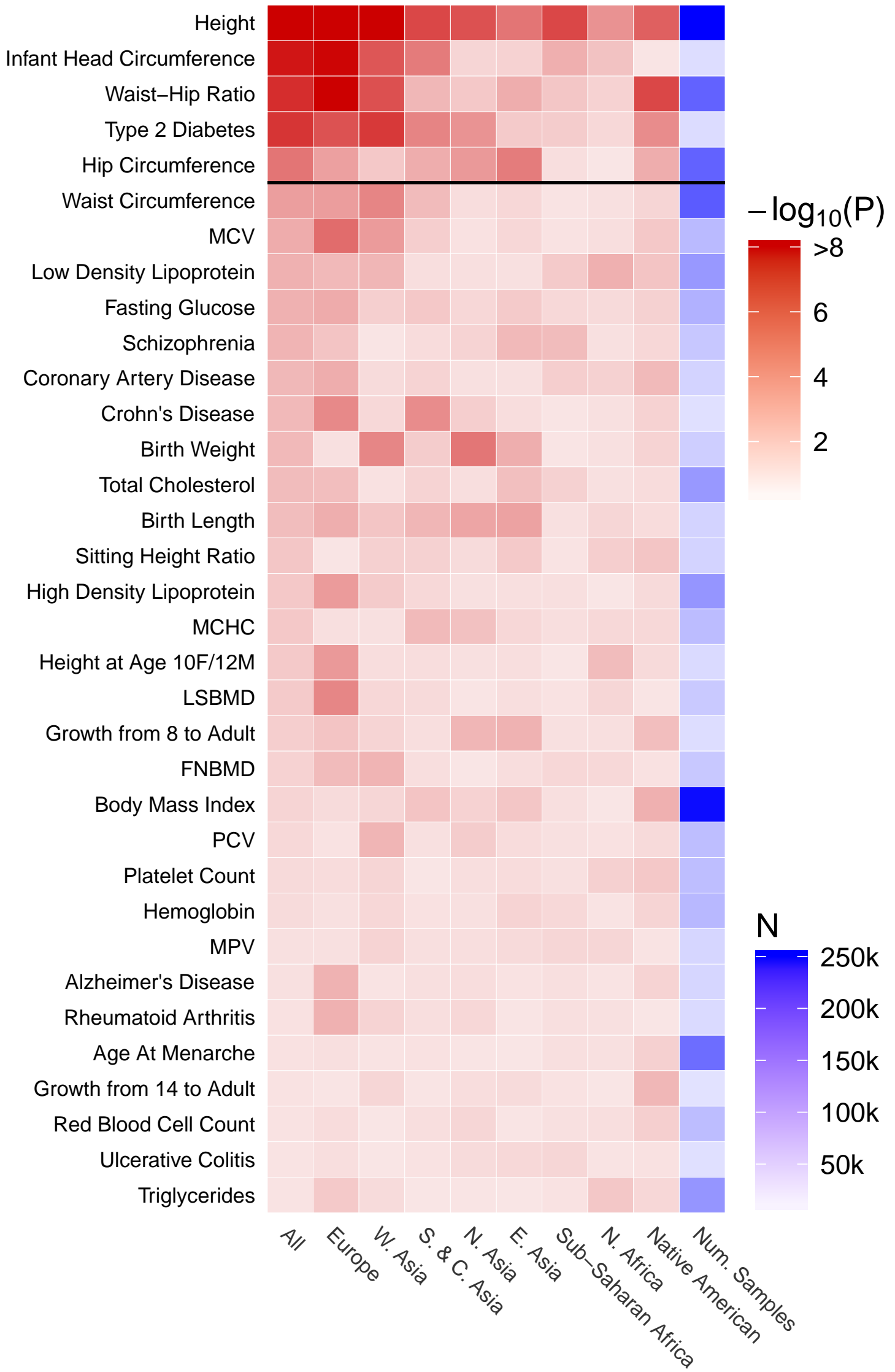

Figure 2: A heatmap showing the $\log 10 \mathrm{p}$-values for the $Q_{X}$ test statistic for over-dispersion of the polygenic scores for a trait among p8pulation samples. The 'All' column gives the pvalue in the combined Human Origin and 1000 Genomes dataset. See $\$ 2$ and $\mathbf{5 1}$ for the definitions of the regional groupings. Each subsequent column gives the score in each geographic sub-region. MCV: Mean red blood cell volume; MCHC: Mean cell hemoglobin concentration; LSBMD: Lumbar spine bone mineral density; FNBMD: Femoral neck bone mineral density; PCV: Packed red blood cell volume; MPV: Mean platelet volume. 


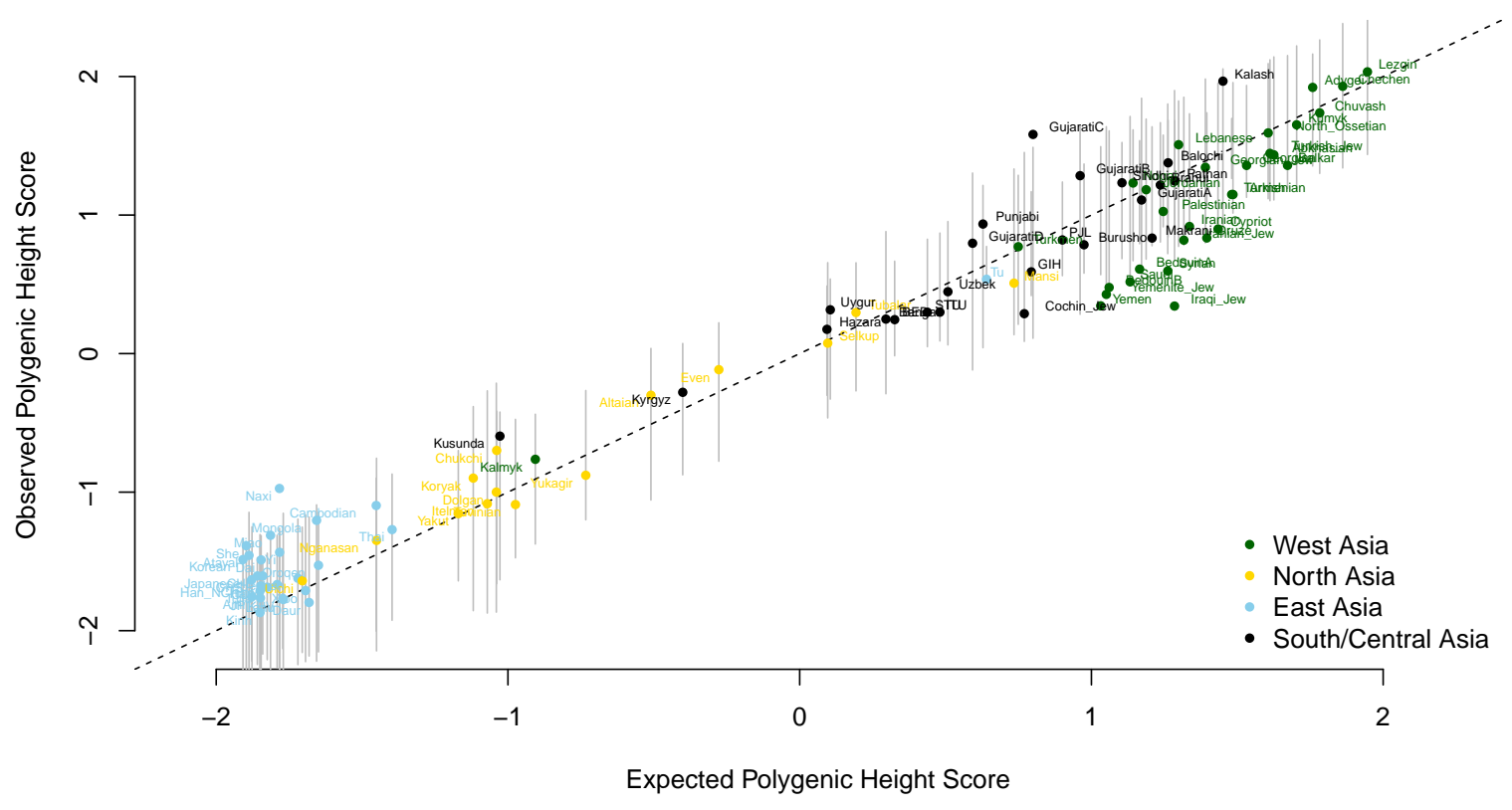

Figure 3: Polygenic height scores in Asia are well-predicted by a model conditioned on European height scores, consistent with selection occurring in a shared ancestral population. An individual population sample's position along the $\mathrm{x}$ axis gives the genetic height score predicted on the basis of scores observed in Europe and their relatedness to the European samples, whereas their position along the y axis gives the true polygenic height score (see Methods for statistical details). The dashed line gives the one-to-one line along which all populations would fall if the predictions were perfectly accurate, whereas the vertical gray lines give population-specific $95 \%$ confidence intervals under genetic drift. 


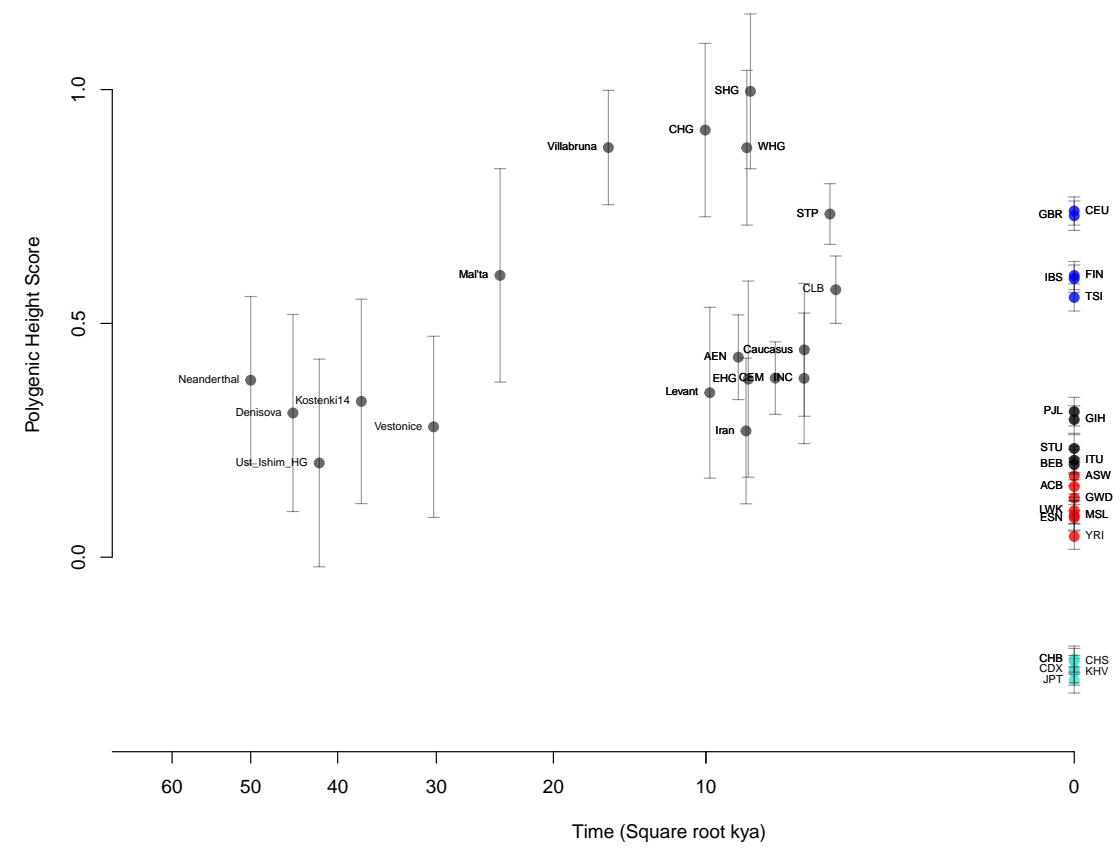

(a)

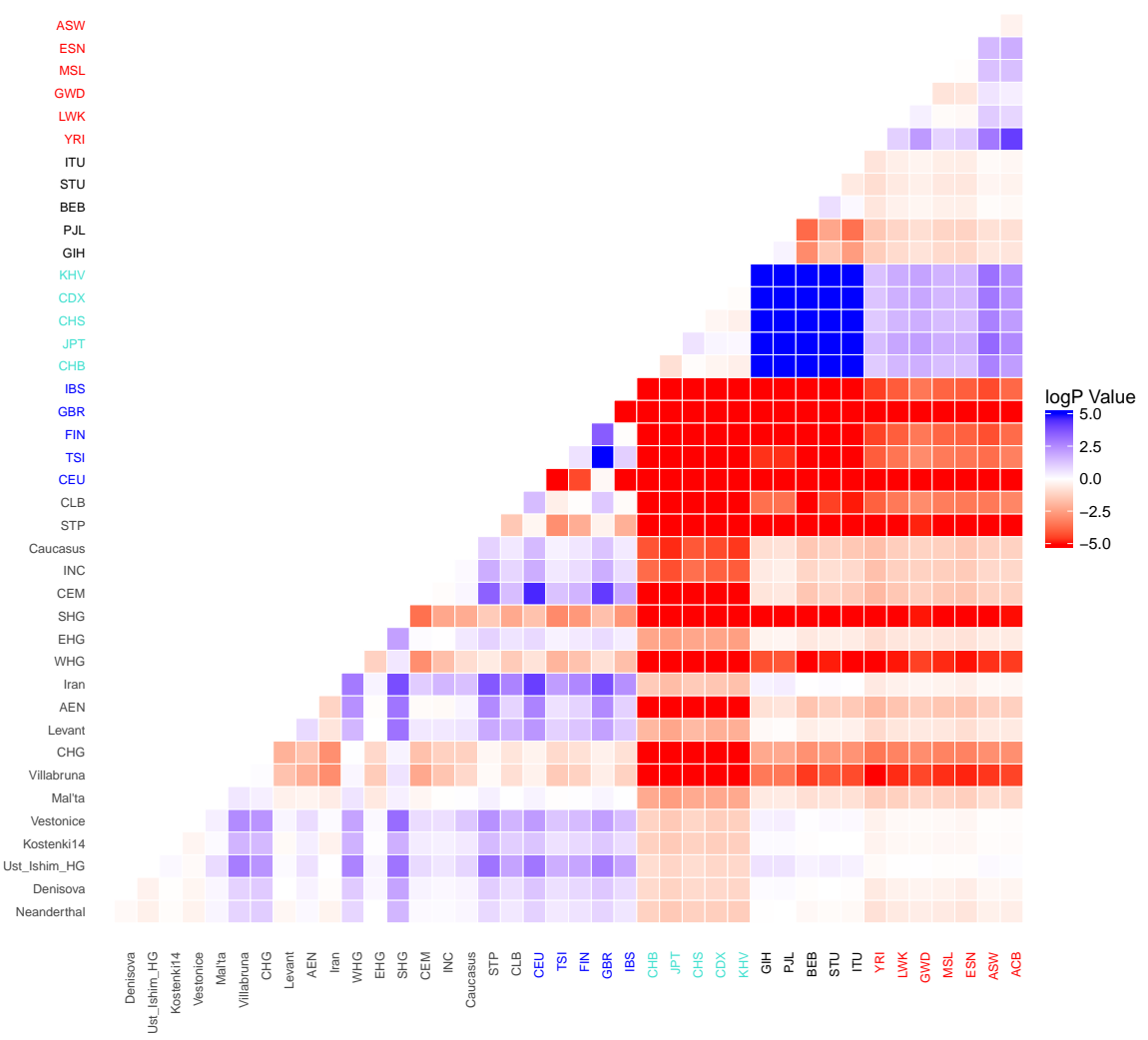

(b)

Figure 4: A) Polygenic height scores for ancient and the modern 1000 genomes population samples. . Each dot show the mean polygenic score for the labeled sample, and the error bars give the $95 \%$ confidence interval. The $\mathrm{x}$ coordinate of each sample is positioned at the mean of the calBP dates for the samples, plotted using a square root transfor to help visualize the spread of ancient populations. AEN, Anatolian Neolithic; WHG, Western hunter-gatherer; CEM, central European Early and Middle Neolithic; INC, Iberian Neolithic and Chalcolithic; CLB, central European Late Neolithic and Bronze Age; STP, steppe. B) A heat map of $\log 10$ p-values for pairwise $Q_{X}$ tests, the p-values are signed by the difference in polygenic score (shades of red denotes the row sample having higher polygenic score than the column sample, and blue the converse) 

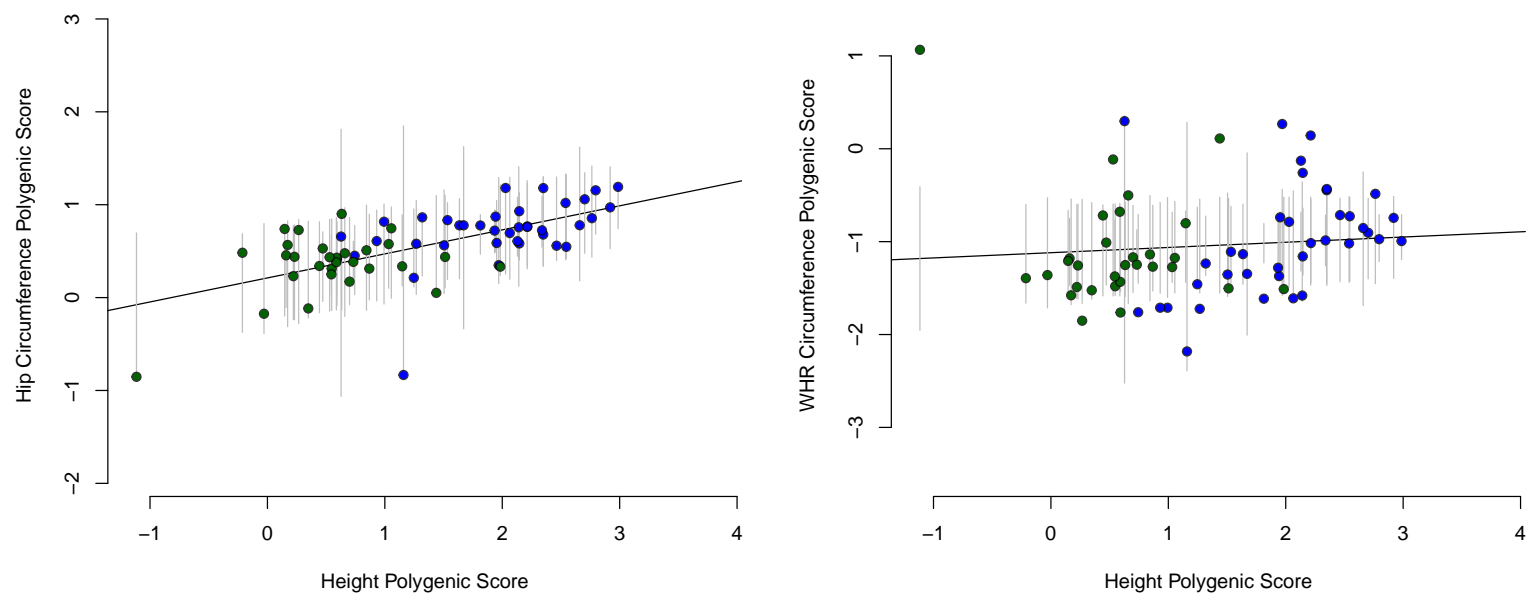

Figure 5: The overdispersion of genetic HIP scores among populations can be explained as a correlated response to selection on height, but such an effect cannot explain the signal of selection on the WHR polygenic scores. A) The observed polygenic HIP score (y axis) plotted against the height polygenic scores (x axis). We show only Western Eurasian population samples (blue dots: Europe; green dots: West Asia), as it is these samples which drive the majority of the signal. The line gives the best prediction for each sample's polygenic HIP score according to the model of a correlated response to selection on height. Vertical lines give the $95 \%$ confidence interval of this prediction for each sample under this model. Most populations' polygenic HIP scores lie within their confidence intervals, consistent with our failure to reject this conditional null model (main text). B) The same as A but now giving polygenic WHR scores rather than HIP. Note that for many populations the WHR scores lie outside of their 95\% CI predictions based on genetic drift and correlated selection on height alone, consistent with the inability of this model to fully capture variation in polygenic WHR scores (main text) 

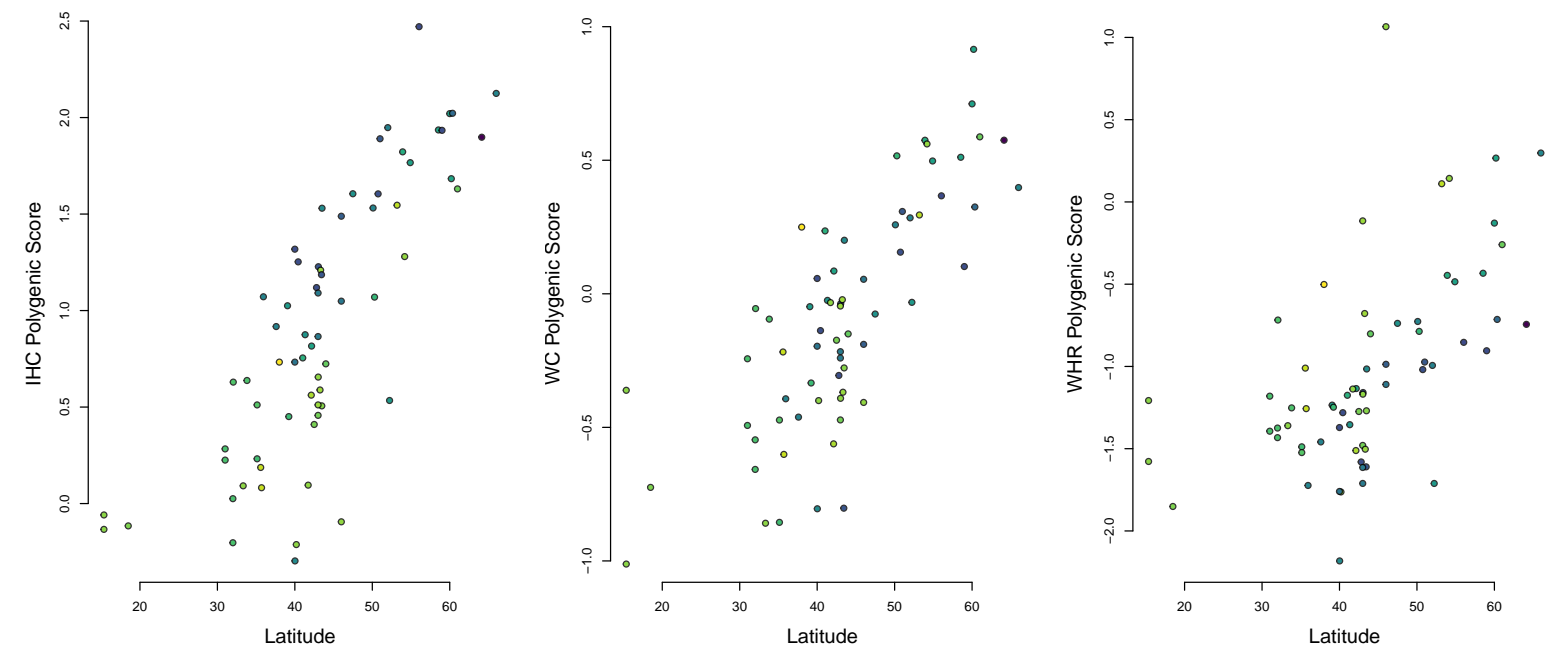

Figure 6: Genetic IHC, WC, and WHR score plotted against Latitude for the Western Eurasian population samples. The points are colored East to West (blue to yellow). 
bioRxiv preprint doi: https://doi.org/10.1101/167551; this version posted April 18, 2019. The copyright holder for this preprint (which was not certified by peer review) is the author/funder, who has granted bioRxiv a license to display the preprint in perpetuity. It is made available under aCC-BY-NC 4.0 International license.

\section{S1 Supplementary material}


bioRxiv preprint doi: https://doi.org/10.1101/167551; this version posted April 18, 2019. The copyright holder for this preprint (which was

\begin{tabular}{|c|c|c|c|}
\hline Trait & Abbrev & Study & Sample size* \\
\hline Age At Menarche & AAM & Perry et al $(2014)^{64}$ & 133 \\
\hline Alzheimer's Disease & $\mathrm{AD}$ & Lambert et al $(2013)^{65}$ & $17 / 37$ \\
\hline Birth Length & $\mathrm{BL}$ & van der Valk et al $(2014)^{66}$ & 22 \\
\hline Birth Weight & BW & Hirokoshi et al $(2013)^{67}$ & 27 \\
\hline BMI (2015) & BMI & Locke et al $(2015)^{68}$ & 240 \\
\hline Coronary Artery Disease & CAD & Schunkert et al $(2011)^{69}$ & $22 / 65$ \\
\hline Crohn's Disease & CD & Jostins et al $(2012)^{70}$ & $6 / 15$ \\
\hline Fasting Glucose & FG & Manning et al $(2012)^{71}$ & 58 \\
\hline Femoral Neck Bone Mineral Density & FNBMD & Estrada et al $(2012)^{72}$ & 33 \\
\hline Hemoglobin & $\mathrm{HB}$ & van der Harst et al $(2012)^{73}$ & 51 \\
\hline High-density lipoproteins & HDL & Teslovich et al $(2010)^{74}$ & 89 \\
\hline Height $(2014)$ & HEIGHT & Wood et al $(2014)^{75}$ & 253 \\
\hline Height at age $10(\mathrm{~F}) / 12(\mathrm{M})$ & Height10F12M & Cousminer et al $(2013)^{76}$ & 14 \\
\hline Hip Circumference (both sexes) & HIP & Shungin et al $(2015)^{77}$ & 169 \\
\hline Hip Circumference adjusted for BMI (both sexes) & HIPadjBMI & Shungin et al $(2015)^{77}$ & 164 \\
\hline Infant Head Circumference & $\mathrm{IHC}$ & Taal et al $(2012)^{78}$ & 10 \\
\hline Low-density lipoproteins & LDL & Teslovich et al $(2010)^{74}$ & 85 \\
\hline Lumbar Spine Bone Mineral Density & LSBMD & Estrada et al $(2012)^{72}$ & 32 \\
\hline Mean cell hemoglobin concentration & $\mathrm{MCHC}$ & van der Harst et al $(2012)^{73}$ & 46 \\
\hline Mean red blood cell volume & $\mathrm{MCV}$ & var der Harst et al $(2012)^{73}$ & 48 \\
\hline Mean platelet volume & MPV & Geiger et al $(2011)^{79}$ & 17 \\
\hline Packed red blood cell volume & $\mathrm{PCV}$ & var der Harst $(2012)^{73}$ & 44 \\
\hline Growth from age 14 to adulthood & PeakGrowthVel14A & Cousminer et al $(2013)^{76}$ & 4 \\
\hline Platelet count & PLT & Geiger et al $(2011)^{79}$ & 44 \\
\hline Growth from age 8 to adulthood & PubertalGrowth8A & Cousminer et al $(2013)^{76}$ & 11 \\
\hline Rheumatoid arthritis & $\mathrm{RA}$ & Okada et al $(2014)^{80}$ & $14 / 44$ \\
\hline Red blood cell count & $\mathrm{RBC}$ & var der Harst $(2012)^{73}$ & 45 \\
\hline Schizophrenia & $\mathrm{SCZ}$ & Ripke et al $(2014)^{81}$ & $34 / 46$ \\
\hline Sitting height ratio & SHR & Chan et al $(2015)^{82}$ & 22 \\
\hline Type 2 Diabetes & $\mathrm{T} 2 \mathrm{D}$ & Morris et al $(2012)^{83}$ & $12 / 57$ \\
\hline Total Cholesterol & $\mathrm{TC}$ & Teslovich et al $(2010)^{74}$ & 89 \\
\hline Triglycerides & TG & Teslovich et al $(2010)^{74}$ & 86 \\
\hline Ulcerative Colitis & $\mathrm{UC}$ & Jostins et al $(2012)^{70}$ & $7 / 21$ \\
\hline Waist Circumference & $\mathrm{WC}$ & Shungin et al $(2015)^{77}$ & 183 \\
\hline Waist Circumference adjusted for BMI & WCadjBMI & Shungin et al $(2015)^{77}$ & 176 \\
\hline Waist-Hip Ratio & WHR & Shungin et al $(2015)^{77}$ & 166 \\
\hline Waist-Hip Ratio adjusted for BMI & WHRadjBMI & Shungin et al $(2015)^{77}$ & 143 \\
\hline
\end{tabular}

Table S1: A list of all of the datasets tested (including those not directly mentioned in the main text), with citations for each study. ${ }^{*}$ For case-control study sample sizes are given as Number of Cases/Number of Controls.

Table S2: A list of all population samples included in our analysis, along with the number of individuals per sample, and our geographic region assignment for each population.

Table S3: A table of the $\log 10 \mathrm{p}$-values for the $Q_{X}$ test statistic for over-dispersion of the polygenic scores for a trait among population samples. The 'All' column gives the p-value in the combined Human Origin and 1000 Genomes dataset. See $\mathbf{5 2}$ and $\mathbf{5 1}$ for the regional definition for the definitions of the regional groupings. Each subsequent column gives the score in each geographic sub-region. MCV: Mean red blood cell volume; MCHC: Mean cell hemoglobin concentration; LSBMD: Lumbar spine bone mineral density; FNBMD: Femoral neck bone mineral density; PCV: Packed red blood cell volume; MPV: Mean platelet volume. Note that this table includes HIP, WC, and WHR adjusted for BMI, in addition to the 42 traits shown in Figure $\boldsymbol{Z}$. These three additional traits were included to followup on the selection signals on HIP, WC, and WHR polygenic scores. 
Table S4: Bivariate tests for evidence of correlated selection. Each row gives the results of a conditional $Q_{X}$ test for evidence that a signal of selection in one trait can be explained as a correlated response to selection on another. Each row corresponds to a choice of two traits (one selected, one not), and a geographic region without which the test was run. The genetic correlation listed is that which gave the least significant $\mathrm{p}$ value (i.e. the most conservative test).

Table S5: Conditional region tests. Each row gives a particular combination of trait, test region, and conditioned region, and presents the $Q_{X}$ statistics and associated p values for that test.

\begin{tabular}{|l|l|l|l|l|l|l|}
\hline & CEU-CDX & TSI-CDX & YRI-CDX & TSI-CEU & YRI-CEU & YRI-TSI \\
\hline Height & 0.05728 & 0.04136 & 0.01136 & -0.01591 & -0.04591 & -0.03 \\
HIP & 0.02559 & 0.02137 & 0.02317 & -0.00422 & -0.00242 & 0.0018 \\
WC & 0.00689 & 0.00265 & 0.01357 & -0.00423 & 0.00668 & 0.01092 \\
WHR & -0.01993 & -0.02677 & -0.00357 & -0.00684 & 0.01635 & 0.0232 \\
IHC & 0.02831 & 0.01988 & -0.00635 & -0.00843 & -0.03466 & -0.02623 \\
T2D & -0.02939 & -0.02377 & 0.00138 & 0.00562 & 0.03077 & 0.02515 \\
\hline
\end{tabular}

Table S6: Average allele frequency differences in the trait increasing allele between a few example populations. This table simply demonstrates that even for phenotypes with very strong differentiation at the polygenic value level, these differences are caused by relatively small average shifts spread across many loci

\begin{tabular}{|l|l|l|}
\hline Cluster & Full Name & Sample size (n) \\
\hline Afanasievo & & 5 \\
Anatolia Neolithic & & 24 \\
Andronovo & & 3 \\
Armenia Chalcolithic & Bell Beaker Late Neolithic & 5 \\
Bell Beaker LN & Central European Late Neolithic and Bronze Age & 17 \\
Central LNBA & 35 \\
Central MN & Central European Middle Neolithic & 6 \\
CHG & Caucasus Hunter Gatherers & 2 \\
Hungary BA & Hungary Bronze Age & 12 \\
Hungary EN & Hungary Early Neolithic & 10 \\
Iberia EN & Iberia Early Neolithic & 4 \\
Iceman & Linearbandkeramik Early Neolithic & 1 \\
LBK EN & & 15 \\
Poltavka & & 7 \\
Sintashta & & 5 \\
Srubnaya & Western European hunter-gatherers & 12 \\
WHG & & 6 \\
Yamnaya Kalmykia & & 9 \\
Yamnaya Samara & & \\
\hline
\end{tabular}

Table S7: A list of all of the ancient samples included in our analysis, along with the number of individuals per sample.

Table S8: A table of all of the Pairwise $Q_{X}$ test statistics and p-values for the comparisons of the 19 ancient and modern Eurasian $1 \mathrm{~kg}$ populations. 


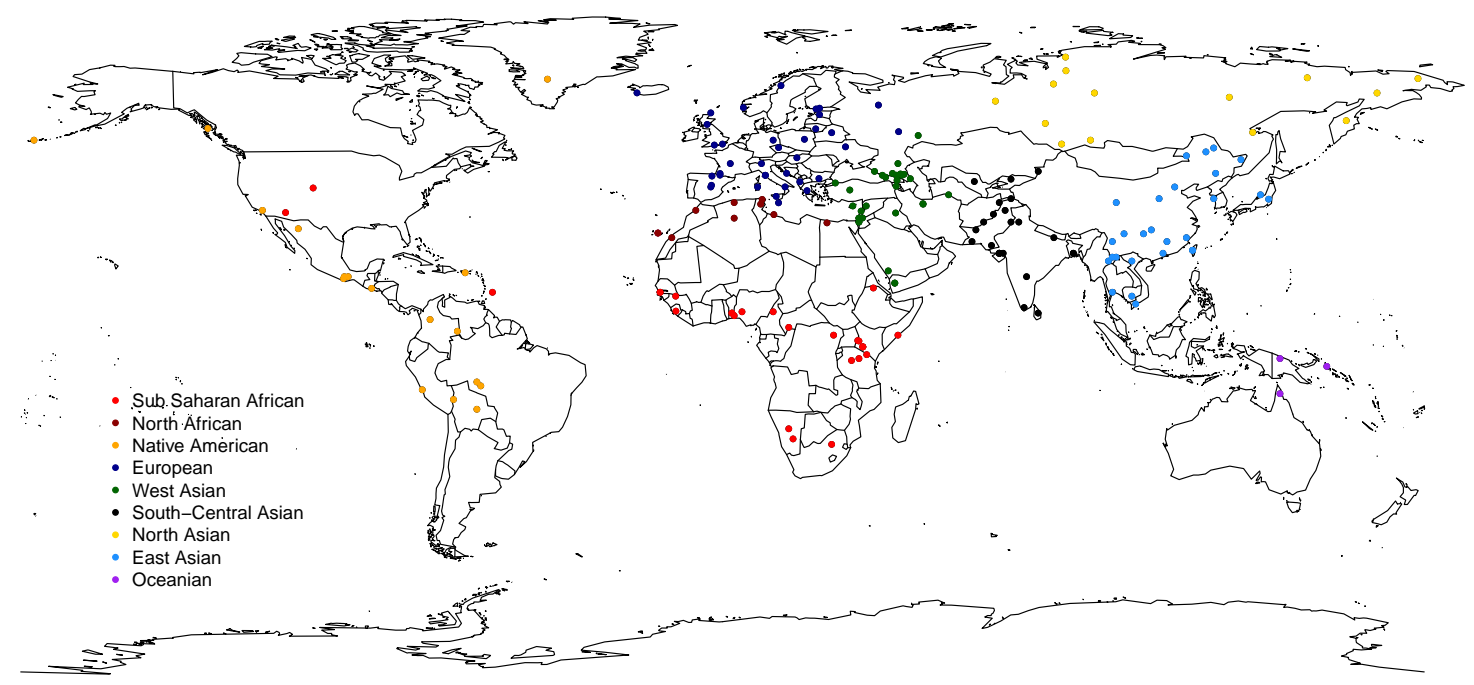

Figure S1: A map showing the locations of all 187 populations with each population colored according to a set of regional labels. Regional groupings were determined via a combination of geography and ancestry.

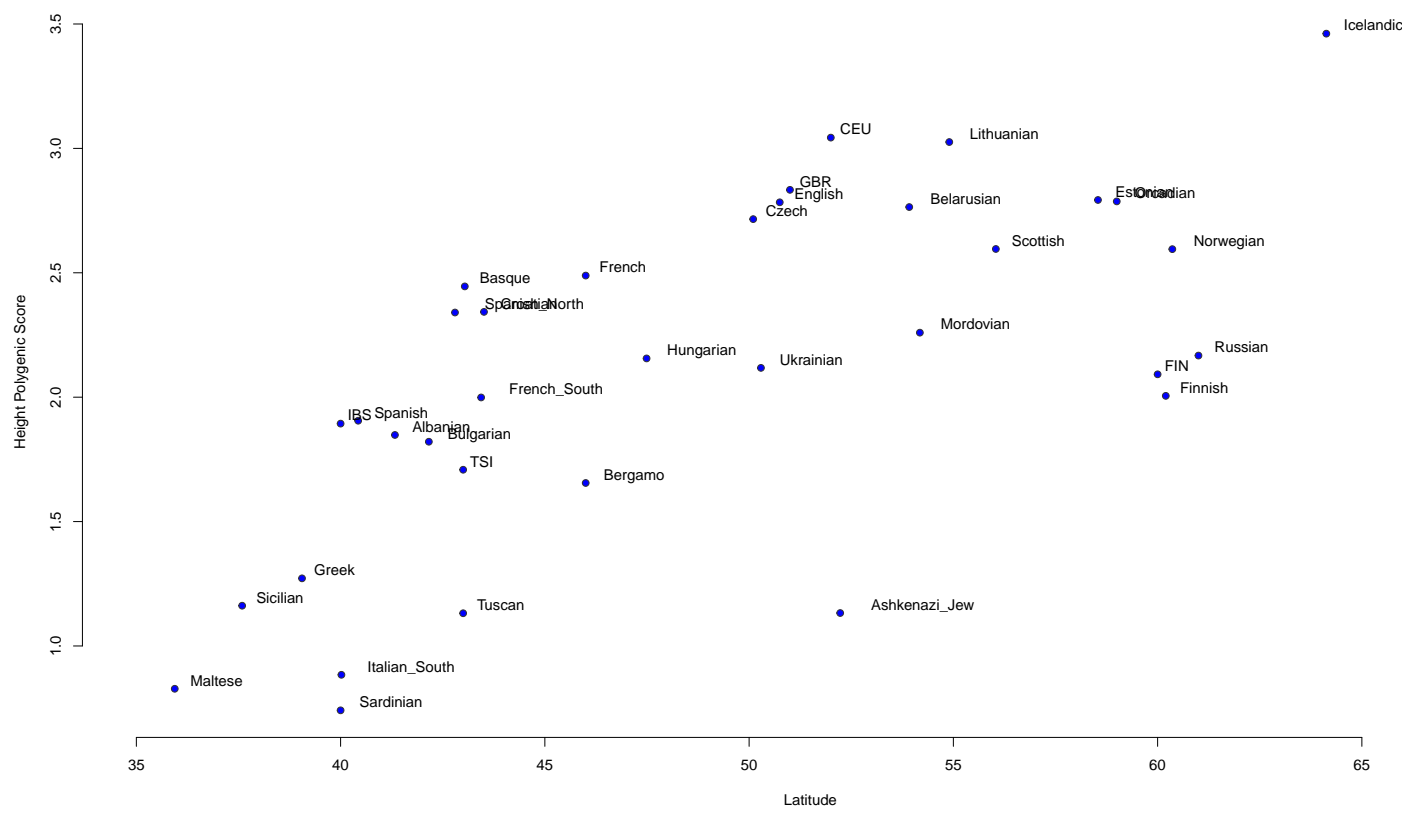

Figure S2: Polygenic scores for height within Europe plotted against latitude. This relationship is strongly significant even after controlling for population structure $\left(p=6.3 \times 10^{-6}\right)$, and represents our replication of previously reported latitudinal clines for height within Europe ${ }^{17,18,19,20,36}$ 

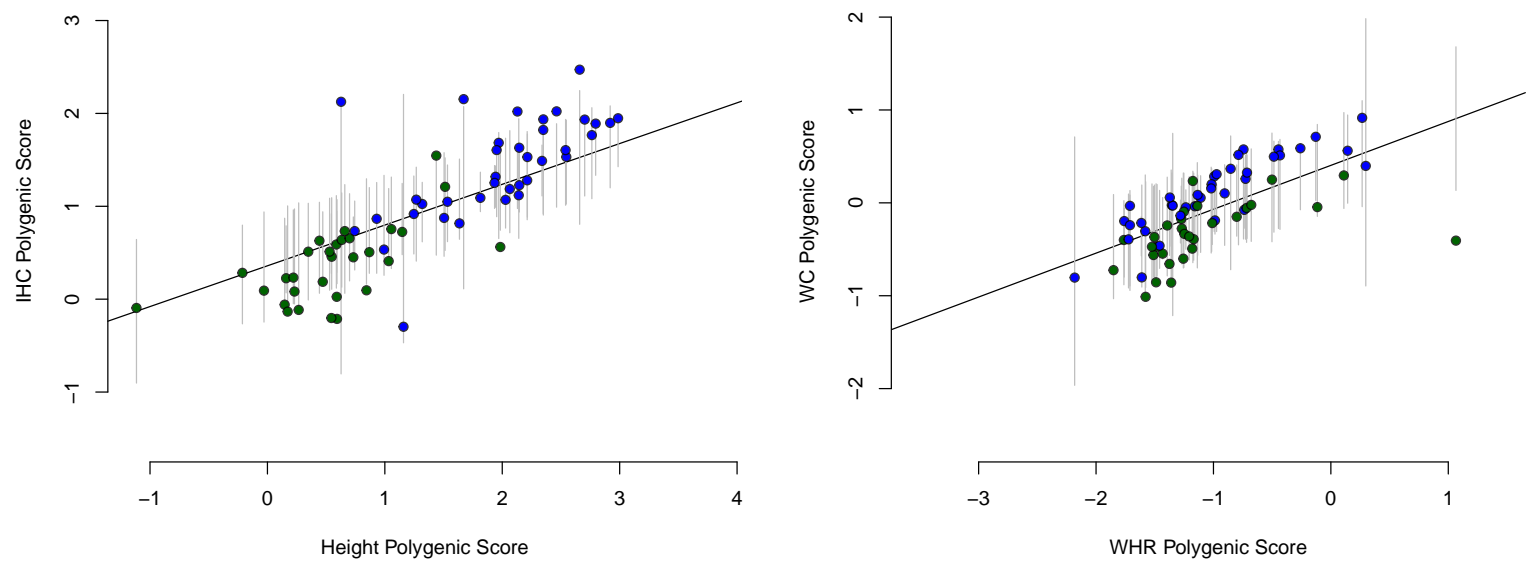

Figure S3: Left) Polygenic scores for IHC plotted against scores for height. Solid line gives the best prediction of IHC given height. Vertical grey lines give 95\% confidence interval for each population. Note that a number of populations fall outside their error bars, consistent with the fact that we reject a neutral model for the evolution of IHC given height (see main text). Right) Same plot but using WHR to predict WC. Note that in this case, most populations fall well within their error bars, in line with the fact that WHR can adequately explain WC in our conditional $Q_{X}$ test. 


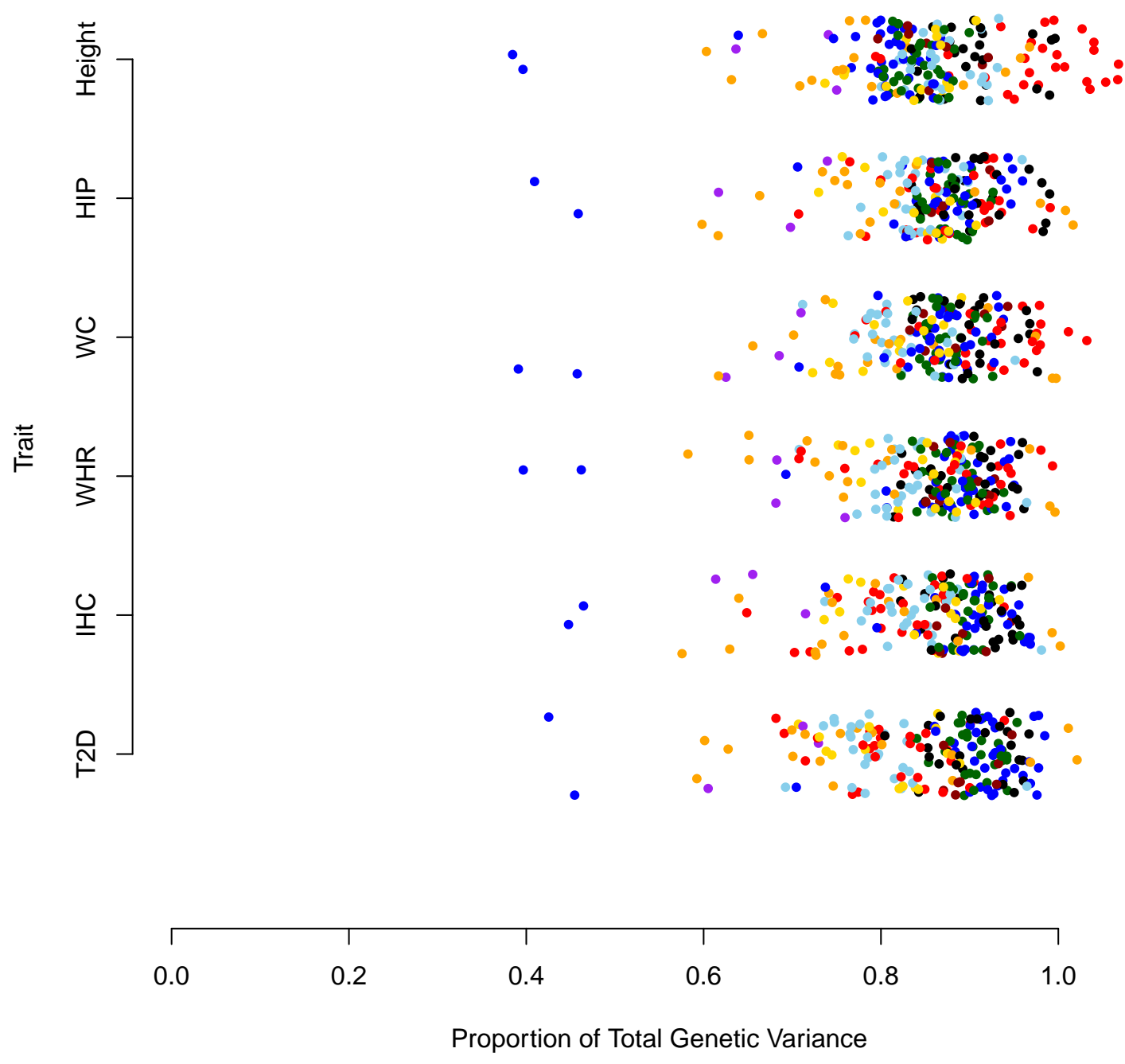

Figure S4: For each of the six traits showing evidence of selection, we show the proportion of total genetic variance for polygenic scores (based on Hardy-Weinberg and linkage equilibrium expectations) present within each population. Color scheme is the same as in figure S]. Note that some of the variation among populations is due to differences in sample size, e.g. the two European (blue) populations with strongly reduced variance for each trait have only a single individual per sample. 


\section{S1.1 Environmental Correlation Tests}

We tested for unusually strong correlations between the polygenic scores for a given trait and an environmental or geographic (hereafter "environmental") variable as follows. Let $\vec{W}$ represent the vector of environmental variables recorded for each of the populations in our dataset. Now define $\vec{Y}$ to be a mean centered and standardized version of $\vec{W}$

$$
\vec{Y}=\frac{\vec{W}-\operatorname{mean}(\vec{W})}{\operatorname{sd}(\vec{W})} .
$$

Next, recall that we have assumed that

$$
\vec{Z} \sim M V N\left(\mu, V_{A} \mathbf{F}\right)
$$

under the null model.

Now, let $\mathbf{C}$ be the Cholesky decomposition (or any other square root) of $\mathbf{F}$, such that

$$
\mathbf{F}=\mathbf{C C}^{T}
$$

We next transform $\vec{Z}$

$$
\vec{X}=\frac{\mathbf{C}^{-1} \vec{Z}}{\sqrt{V_{A}}}
$$

such that $\vec{X} \sim N(0,1)$ under the null (which each element of $\vec{X}$ independent), but will retain information about any excess correlation with an environmental variable that is not predicted by drift and shared population history alone.

In order to test for such correlation, we must also transform the environmental variable

$$
\vec{Y}^{*}=\mathbf{C}^{-1} \vec{Y}
$$

We then take the pearson product moment correlation between $\vec{X}$ and $\vec{Y}^{*}$ as a test statistic for the correlation between the polygenic scores and the environmental variable. Notably, because the test is performed in a rotated coordinate system that removes the effect of population structure, the test will have higher power and a lower false positive rate than a naive test of the untransformed polygenic scores. As with all of our other tests, in order to test for significance, we compare to a null distribution generated by calculating null sets of polygenic scores assembled from resampled SNPs matched for derived allele frequency to the CEU population sample so as to mimic the effects of the GWAS ascertainment. 


\section{S1.2 Eigendecomposition of $Q_{X}$ statistic}

In constructing our empirical null statistic we make use of the fact that we can break the $Q_{X}$ statistic down into the projection of the polygenic scores along each of the eigen-vectors of the matrix $\mathbf{F}$. Consider that our test statistic is given by

$$
Q_{X}=\frac{\vec{Z}^{T} \mathbf{F}^{-1} \vec{Z}}{V_{A}}
$$

Now, we write the eigendecomposition of $\mathbf{F}$ as

$$
\mathbf{F}=\mathbf{U} \boldsymbol{\Lambda} \mathbf{U}^{T}
$$

where $\mathbf{U}$ is a matrix containing the eigenvectors $(\vec{U})$ of $\mathbf{F}$ as its columns, and $\boldsymbol{\Lambda}$ is a matrix with the eigenvalues $(\lambda)$ on the diagonal and zeroes elsewhere. For each eigenvector, we can define a statistic

$$
q_{U}(i)=\frac{\vec{U}_{i}^{T} \vec{Z}}{\sqrt{\lambda_{i} V_{A}}}
$$

which is the slope of the regression of polygenic scores on the $i^{t h}$ eigenvector $\left(\vec{u}_{i} \vec{Z}\right)$ divided by the standard deviation of this regression coefficient under the null $\left(\lambda_{i} \sqrt{V_{A}}\right)$. By the definitions of the multivariate normal distribution and the eigenvalue decomposition, this statistic has mean zero, a variance of one, and is linearly independent from all other such statistics $q_{u}(j)$ for $j \neq i$. Note that the square of this statistic,

$$
Q_{U}(i)=q_{U}(i)^{2}
$$

has a $\chi_{1}^{2}$ distribution under the null hypothesis, and because of their independence, our global test statistic $Q_{X}$ can be written as a sum of the $Q_{u}(i)$ s:

$$
\begin{aligned}
Q_{X} & =\frac{\vec{Z}^{T} \mathbf{F}^{-1} \vec{Z}}{V_{A}} \\
& =\frac{\vec{Z}^{T} \mathbf{U} \boldsymbol{\Lambda}^{-1} \mathbf{U}^{T} \vec{Z}}{V_{A}} \\
& =\sum_{i} \frac{\left(\vec{U}_{i}^{T} \vec{Z}\right)^{2}}{V_{A} \lambda_{i}} \\
& =\sum_{i} q_{U}(i)^{2} \\
& =\sum_{i} Q_{U}(i)
\end{aligned}
$$




\section{S1.3 Empirical Null}

The MVN model of drift is generally justified by supposing that we are told the frequency of an allele in a given population, and then asked to predict the joint distribution of allele frequencies across multiple descendant populations after a relatively small amount of neutral evolution. In this case, the MVN model is generally a good approximation to the diffusion (see previous work ${ }^{31,84,18}$ for more extended descriptions of this approximation).

However, consider an alternative case where instead of being told the frequency of the allele in the ancestral population, we are told the frequency in a single one of the descendant populations, and we are also told that the allele has a single mutational origin and we are told which allele is derived and which is ancestral. This knowledge alone is sufficient to violate the assumptions of the MVN model, as it must be the case that, looking backward in time from the present, the frequency of the derived allele decreases on average back until we reach the mutation which created it. Playing the tape forward in time, it is then clear that the expected change in allele frequency along the lineage leading to the conditioned upon population is not zero. Indeed, the effect is essentially a form of the "fictitious selection" described by Zhao and colleagues, ${ }^{85}$ that arises for alleles (neutral or otherwise) whose fate (forward or backward in time) is conditioned on.

Our case more closely resembles the latter example, as GWAS loci are ascertained in a particular present day population, and they must be at sufficiently intermediate frequencies in order to be detected. We might therefore fear that the MVN does not strictly hold. Because positive signals in our test are generally created when the sign of an allele's effect on the trait is predictive of its distribution among populations (see previous work by Kremer and Le Corre ${ }^{86,87}$ and ourselves ${ }^{18}$ for more extended discussions of this fact), we are most concerned about this problem when there is a correlation (within our set of GWAS loci) between the sign of an allele's effect on the trait and whether or not it is derived or ancestral.

In figure $\mathbf{5 . 5}$, we show the the $q_{U}(i)$ statistic for the schizophrenia dataset for the top 30 eigenvectors of the population genetic covariance matrix (black dots), compared with an empirical null distribution for each $q_{U}(i)$ statistic that is constructed by resampling SNPs which matched the derived allele frequency of the true associations in the 1000 genomes CEU panel (which we take as a proxy for the GWAS population). The exact procedure is detailed at the end of this section. Strikingly, we observe that for some of the eigenvectors (e.g. 1,2 and 4), the ascertainment matched empirical null distribution has a mean that is shifted away from zero, and toward the direction of the observed true $q_{U}(i)$ statistic for that eigenvector.

This demonstrates that at least some of the signal we naively observe for schizophrenia has been created by the GWAS ascertainment procedure, and does not actually reflect the action of natural selection. Fortunately, these eigenvector statistics (i.e. the $q_{U}(i)$ ) offer an attractive route to controlling for these ascertainment effects. We define a recentered and standardized version of these 


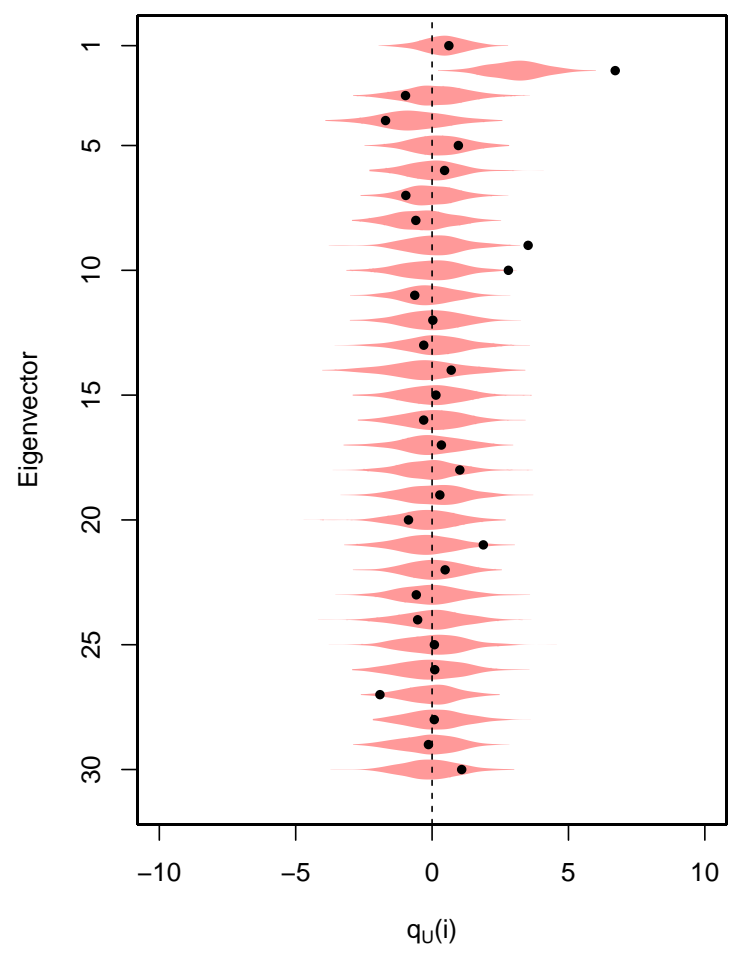

Figure S5: Violin plot of the eigenvector statistics for Schizophrenia. Each violin shows the empirical frequency matched null distribution for each eigenvector. Black dots give the eigenvector statistics for the true data. Violins which are not centered at zero, or which have variance greater than 1 indicate departures from the neutral null model caused by ascertainment 
eigenvector statistics as

$$
q_{U}^{*}(i)=\frac{q_{U}(i)-\mu_{q_{U}(i)}}{\sigma_{q_{U}(i)}}
$$

where $\mu_{q_{U}(i)}$ is the mean of the empirically resampled null $q_{U}(i)$ statistics, and $\sigma_{q_{U}(i)}$ their standard deviation. This ensures that each $q_{U}(i)$ has mean zero and standard deviation one under the empirically recalibrated null, and we then take as our global test statistic the rescaled $Q_{X}$ statistic

$$
Q_{X}^{*}=\sum_{i} q_{U}^{*}(i)^{2}=\sum_{i} Q_{U}^{*}(i)
$$

which should follow the appropriate $\chi^{2}$ distribution under the empirically calibrated null hypothesis. Throughout the paper we report $\mathrm{p}$ values derived from this empirical null unless otherwise stated.

The two phenotypes most strongly impacted by this ascertainment effect are schizophrenia and type 2 diabetes. For schizophrenia, we find that after applying the recalibrated null the naive p value $\left(p=9.5 \times 10^{-6}\right)$ is reduced substantially $(p=0.018)$, such that in the end the strength of evidence against the null is relatively marginal. For type 2 diabetes, on the other hand, while comparison to the empirical null does reduce the strength of the signal, we still see significant evidence against the null even after comparison to the recalibrated null (naive $p=6.17 \times 10^{-11}$ vs recalibrated $\left.p=1.27 \times 10^{-6}\right)$.

\section{S1.4 Comparison between risk and protective variants for schizophre- nia and type 2 diabetes}

We noticed that the two phenotypes for which the empirically calibrated null deviated most strongly from the naive null were both disease traits (i.e. schizophrenia and type 2 diabetes). This is noteworthy because the loci identified for these phenotypes have been ascertained under the case-control study design, which is known to result in asymmetries in statistical power when the number of cases and controls are not equal (as they seldom are), with lower power to identify low frequency protective alleles than low frequency risk alleles. ${ }^{88}$ We were also concerned that a neutral model may not be an appropriate null for these phenotypes, as being diseases with severe fitness consequences, we might expect a priori that there would be systematic selection against risk increasing alleles and for risk decreasing alleles. Further, the combination of ascertainment bias and systematic selection against disease alleles might generate signals under our test that are real, in the sense that they represent a real long term response to selection on the GWAS loci identified, but may be misleading if interpreted naively as a signal of divergent selection among populations.

To understand how these two effects may have played a role in generating the signals we observe for SCZ and T2D, we separated alleles into two classes on the basis of whether the derived allele is a risk variant or a protective variant. We can then develop qualitative expectations about what sort of patterns we expect to see if either of the above mechanisms (asymmetric power and/or systematic 
selection against disease) are at play. Asymmetric power should have two major consequences. First, increased relative power to detect low frequency risk variants means that on average the most significant variant in a given block should be a derived risk variant greater than $50 \%$ of the time (as on average the rarer of the two alleles at a site will be the derived allele the majority of the time). This is consistent with what we observe for both diseases (T2D: 772 out of 1670 derived variants are protective, two tailed binomial test $\mathrm{p}=0.002213$; SCZ: 699 out of 1496 derived variants are protective, binomial test $\mathrm{p}=0.012121)$. Second, protective variants that are detected should be systematically closer to $50 \%$ frequency than risk variants. Using the CEU as a proxy for the GWAS sample of primarily north-western European ancestry, we observe this for both diseases (T2D: derived protective mean frequency $=35.5 \%$ while derived risk mean frequency $=27.5 \%$; SCZ: derived protective mean frequency $=35.5 \%$, while derived risk mean frequency $=22.6 \%$ ). This asymmetry alone would be sufficient to generate signal in our naive test prior to empirical calibration of the null model, and in our framework would be expected to present as selection for decreased risk in Europeans relative to other populations because derived allele frequencies should be lower in populations genetically distant to the European GWAS population. One way to think about this is that the "fictitious selection" described above due to conditioning in the GWAS is stronger for derived protective variants than for derived risk variants . Other populations' polygenic scores should also show this effect in a manner than depends upon how recently they share ancestry with the population in which the GWAS was done. However, because this effect involves no actual selection, it can be entirely controlled for by recalibration of the empirical null model to condition on the set of derived allele frequencies (as described in our Empirical Null Section).

In Figures $\mathbf{5 6}$ and $\mathbf{5 7}$ we show the observed mean frequency of derived risk and protective alleles in the CEU and YRI population samples. We also show the mean frequency for control derived alleles with matched frequencies in CEU. The lower frequency of the matched derived alleles in YRI clearly shows why a frequency matched null is necessary, as both the matched control protective alleles and the risk alleles have a higher average allele frequency in the CEU than the YRI due to the fictitious selection effect described above. However, both disease traits show some deviation away from the null expectation in these figures, particularly $\mathrm{T} 2 \mathrm{D}$, consistent with the fact that we strongly reject the neutral null model for T2D, but find only marginal signal at best for SCZ after controlling for ascertainment effects via our empirical null.

Our empirical null is based on neutral evolution, whereas we might expect these disease phenotypes to have been selected against a priori and in a similar manner across all populations, suggesting that our neutral model may not be an appropriate null. Adjusting our null expectation to account for the fact that we expect diseases to be systematically selected against is more challenging, and a detailed quantitative understanding is beyond the scope of this paper, but here we develop some qualitative expectations. First, consider a disease trait under constant negative selection, and compare protective alleles ascertained in the CEU (or a closely related sample of European individuals), 
to loci randomly sampled so as to have the same frequency distribution within the CEU. We expect that both the protective alleles and the control alleles should have a higher average allele frequency in the CEU than the YRI due to the fictitious selection effect described above. However, we might expect relatively little difference between protective and control alleles in YRI, as the fact that the protective alleles experience positive selection in the lineage leading to YRI while the controls do not is offset by the fact, given that they have been under positive selection, protective alleles were likely at lower frequency at the time of the split between the ancestors of CEU and YRI. Risk alleles, on the other hand, will have experienced the same fictitious selection in the lineage leading to CEU, but will have been held at lower frequency in the YRI due to selection against the disease. These two patterns are pictured together in figure $\mathbf{5 8} \mathrm{A}$.

However, our results for T2D (and to a lesser extent SCZ) do not match this qualitative expectation from a model of constant selection against the disease. It may be consistent with a model where selection pressures against the disease differ among populations. In the case of differential selection for lower population risk in CEU relative to YRI, we expect a different pattern, where protective alleles should be at systematically lower frequencies in YRI than their matched controls (reflecting their selection upward in frequency within CEU since the two populations split), while risk increasing alleles will tend to be at higher frequencies in YRI than matched controls, reflecting stronger negative selection against these alleles in the ancestors of the CEU than those of the YRI. This pattern is depicted in figure $\mathbf{5 8 \mathrm { B }}$, and closely resembles that observed for T2D. However, a model based framework accounting for both ascertainment and purifying selection against disease will be needed to more fully demonstrate this.

\section{S1.5 Polygenic Height Scores and Ancient DNA}

Overall, the previously detected selection signal of increased polygenic height scores in modern Northern Europeans is replicated in the ancestral Western Eurasian populations, with modern East Asians having the lowest polygenic height scores. This suggests that this signal of genetic height differentiation across Eurasia is old. The Anatolian Neolithic group and Iberian early farmers (Iberia EN, Iberia MN etc.) both show significantly reduced polygenic height scores relative to modern Europeans, that are only somewhat higher than East Asians. The Steppe populations (Andronovo, Srubnaya etc.) shows increased polygenic height scores, which are consistent with signals reported from Mathieson et al., ${ }^{19}$ with one exception for Hunter-Gatherer groups, which in our analysis show a distinct (and strongly statistically significant) increase in polygenic height scores. This increased signal $^{19}$ in the Hunter-Gatherer groups likely reflects the increase in number of height associated loci included (724 vs. 180). In total, this is consistent with the view that the polygenic height score difference we see across Eurasia is old, and that as hypothesized by Mathieson et al. ${ }^{19}$ the modern gradient in polygenic height scores within Europe may be mostly driven by the mixture between 


\section{SCZ_2014}

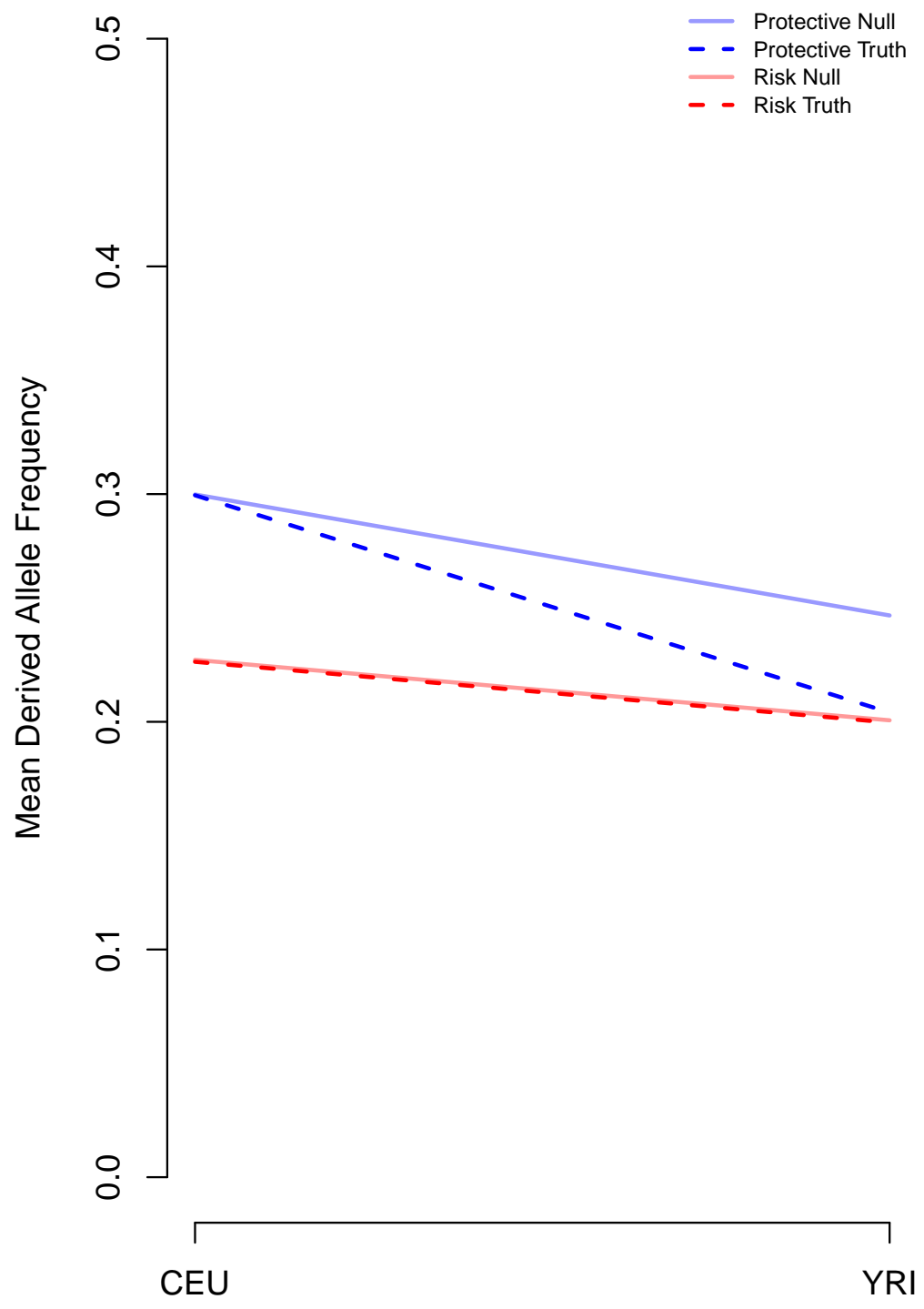

Figure S6: Observed pattern of SCZ risk and protective variants when comparing allele frequencies in CEU and YRI population samples. 
T2D

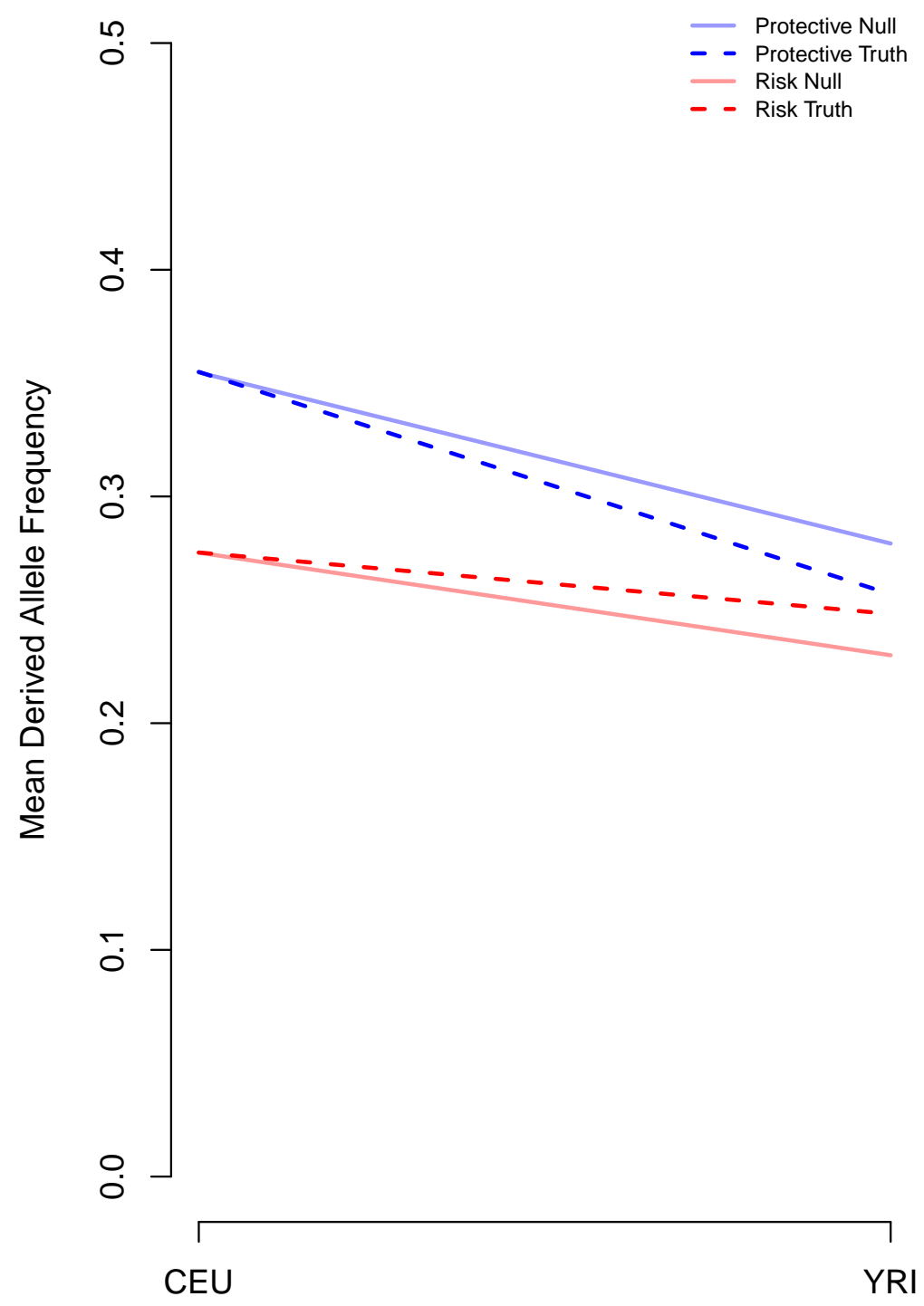

Figure S7: Observed pattern of T2D risk and protective variants when comparing allele frequencies in CEU and YRI population samples. 
Mut Sel Balance Expectation

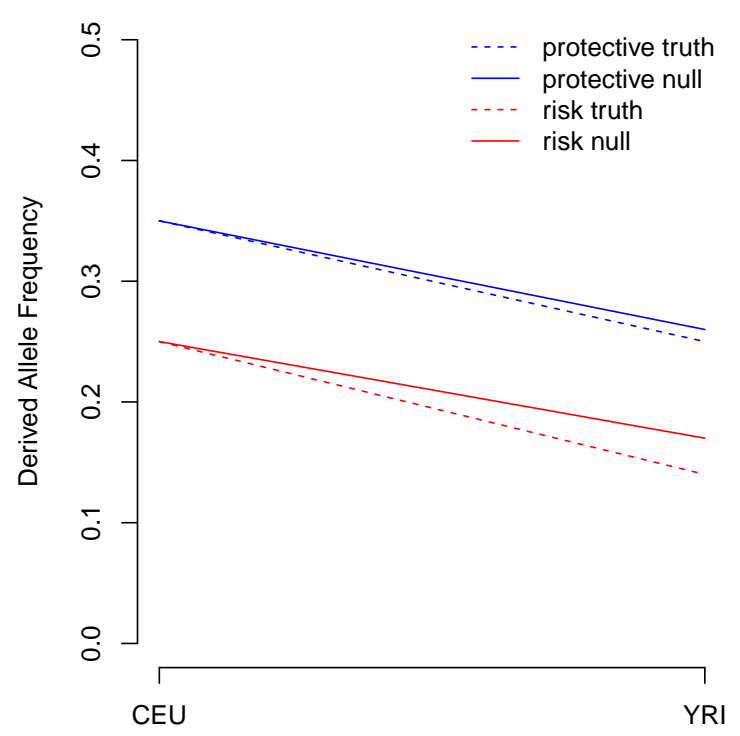

Adaptation in CEU

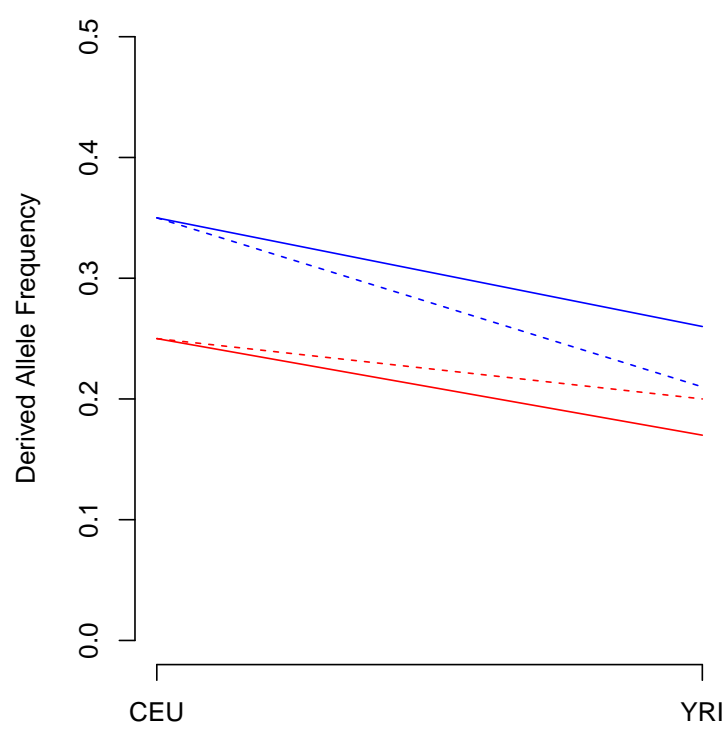

Figure S8: Two hypothetical patterns in the distribution of protective and risk alleles expected for disease traits which are a priori deleterious

ancestral groups who had diverged in polygenic height scores.

To further explore this we looked at the decomposition of European populations by ancient ancestries. Haak et al. $^{43}$ has previously shown that most modern populations with European ancestry can be well described as a two-way mixture of WHG (modeled by Loschbour ancestry) and early Neolithic ancestry (modeled by LBK EN ancestry), followed a third wave of ancestry from the Yamnaya Steppe populations. For each of the European HO panel population samples we extracted the proportions of WHG, early Neolithic ancestry, and Yamnaya ancestry from Haak et al. ${ }^{43}$ (these are reproduced in Figure S.9]). In Figure STOA-C we plot the proportion of each of these ancestries against the polygenic height for each modern European HO population (calculated at our subset of $724 \mathrm{SNPs})$. For comparison to these modern values we also plot, as horizontal lines, the mean polygenic height of the CEM (the analysis cluster containing LBK EN), the WHG (which contains the Loschbour individual), and the STP (which contains the Yamnaya samples).

Much of the variation in ancestry is along the Yamnaya-Early Neolithic axis, with the pearson correlation between Yamnaya and Early Neolithic ancestry being -0.92 across populations. There is somewhat less variation in WHG ancestry among modern European populations, WHG ancestry is corelated with Yamnaya ancestry (a pearson correlation of 0.60) and negatively correlated with Early Neolithic ancestry (a pearson correlation of -0.85). Modern European polygenic height scores are strongly correlated with the Yamnaya-Early Neolithic axis (e.g. pearson correlation of Yamnaya ancestry and polygenic height score is 0.69 , p-value 0.0002 , see also Mathieson ${ }^{89}$ ). To see how well 


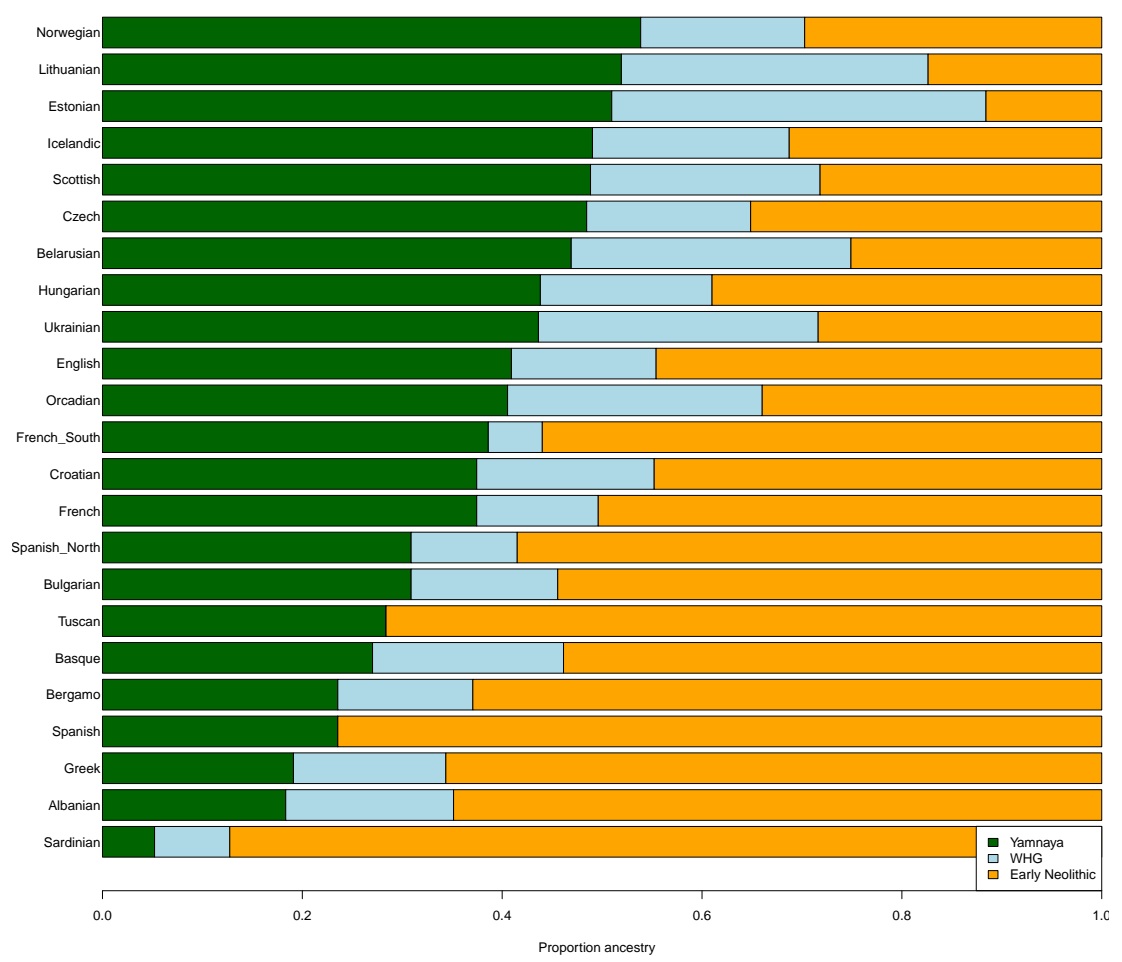

Figure S9: Modern European Admixture proportion estimates taken from Haak et al. ${ }^{43}$

modern European polygenic height scores could be predicted just on the basis of ancient polygenic height scores alone, we predicted each modern population $(i)$ based on its admixture proportions from the Yamnaya $\left(f_{i, Y a m}\right)$, Early Neolithic $\left(f_{i, E N}\right)$, and Western Hunter Gatherer $\left(f_{i, W H G}\right)$ as

$$
Z_{i, \text { pred }}=f_{i, Y a m} Z_{Y a m}+f_{i, E N} Z_{E N}+f_{i, W H G} Z_{W H G}
$$

where for $Z_{Y a m}, Z_{E N}$, and $Z_{W H G}$ we used the polygenic score of the STP, CEM, and WHG respectively (calcuated over our 724 SNPs, calculated as described in main text). In Figure 5T0 we show these predictions plotted against the observed polygenic height scores (for the same SNP set). Overall the prediction works reasonable well. The prediction captures much of the overall height of the European polygenic scores, and the variation among populations. This suggests that the overall level of polygenic scores in modern Europeans is mostly attributable to high polygenic scores of WHG and STP populations, and that variation in polygenic heights is well explained by the differential contributions of early Neolithic, Western Hunter-Gatherers, and Early Neolithic populations to the ancestry of modern populations. While a reasonable fit, many of the observed values fall somewhat above their predictions. This is consistent with Europe-wide selection for increased polygenic height scores since the Yamnaya expansion, or (perhaps more realistically) that one of our source heights is mis-estimated by the ancient proxy. For example, perhaps the Yamnaya population who contributed ancestry to Europeans had a higher polygenic height score than that of our proxy sample. 

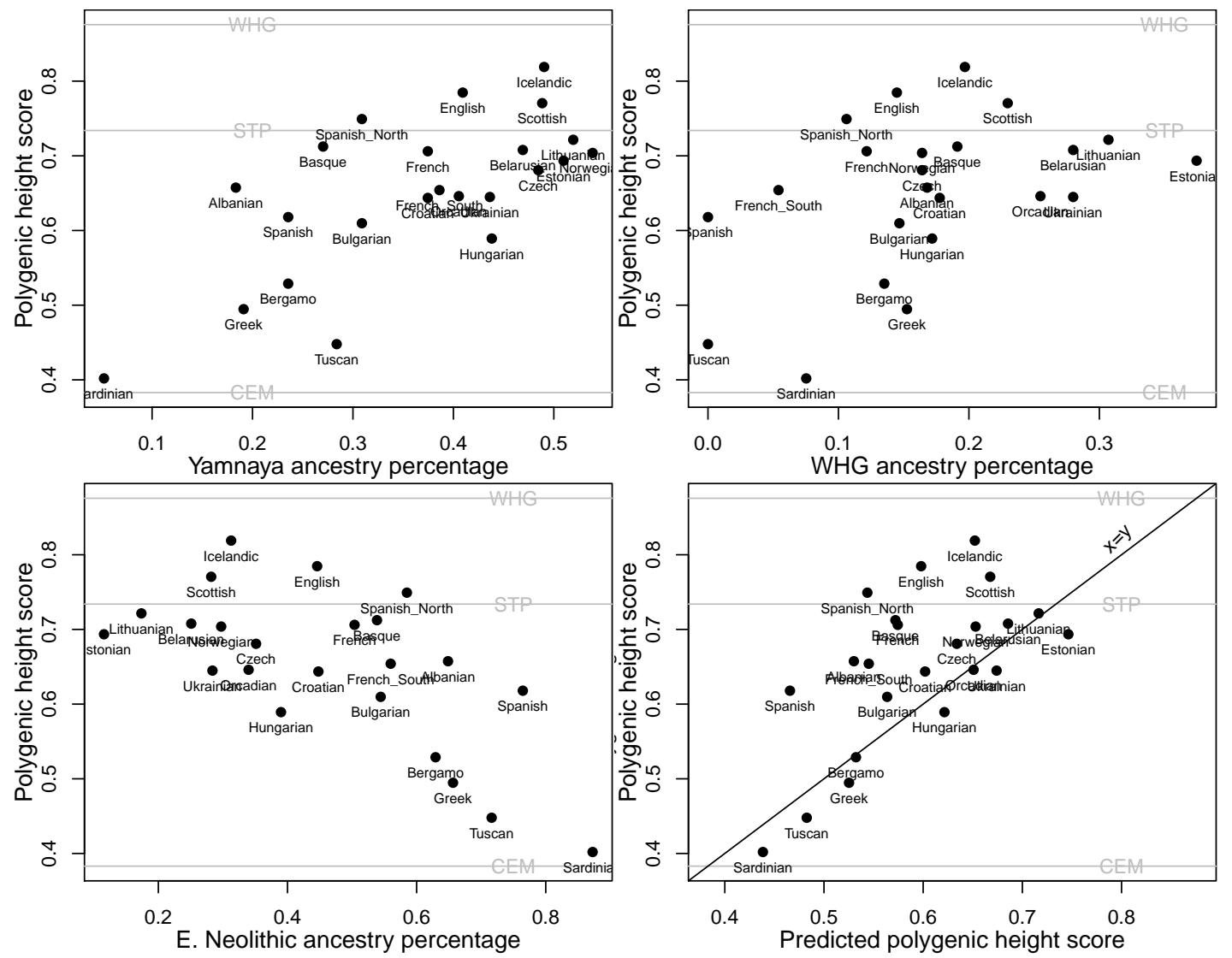

Figure S10: A-C Polygenic height scores for modern European populations plotted against their ancestry proportions for Yamnaya Steppe, WHG, and early Neolithic ancestry. The grey horizontal lines give the polygenic height scores for the WHG, STP, and CEM (central European Early and Middle Neolithic) population samples. D) Polygenic height scores across modern European populations plotted againt a prediction for the scores based on ancient populations. 


\section{S1.6 Two Trait tests}

\section{S1.6.1 Conditional Tests}

If $\vec{Z}_{1}$ and $\vec{Z}_{2}$ are vectors of polygenic scores for two different traits constructed according to equation (四), and the matrix $\mathbf{Z}=\left[\vec{Z}_{1}, \vec{Z}_{2}\right]$ contains these vectors as columns, then under neutrality the distribution of $\mathbf{Z}$ is approximately matrix variate normal

$$
\mathbf{Z} \sim M V N_{M \times 2}(\mu, \mathbf{F}, \mathbf{G})
$$

where the matrix $\mu$ contains the trait specific means, $\mathbf{F}$ gives the covariance structure among rows as in the single trait model, while $\mathbf{G}$ gives the covariance structure among columns. The matrix $\mathbf{G}$ is the canonical among trait genetic covariance matrix, or the "G matrix" of multivariate quantitative genetics ${ }^{47}$ estimated for a population ancestral to all populations in the sample. The diagonal elements of this matrix are given by the $V_{A}$ parameters from above in the single trait model, calculated independently for each trait. Off diagonal elements correspond to the additive genetic covariance between the two traits. In the case where some loci contribute to both traits, and all loci are approximately unlinked, these genetic covariances can be calculated as

$$
g_{12}=C_{A, 12}=\sum_{i} \alpha_{1 i} \alpha_{2 i} \bar{p}_{i}\left(1-\bar{p}_{i}\right)
$$

where $\alpha_{1 i}$ and $\alpha_{2 i}$ are the effects of locus $i$ on traits 1 and 2 respectively. Given this null model for the joint distribution of the two traits, we can construct a conditional model for the distribution of trait 1 , given values observed for trait 2 , as

$$
\begin{aligned}
\vec{Z}_{1} & \sim M V N\left(\xi, V_{A C} \mathbf{F}\right) \\
\xi & =\mu_{1}+\frac{C_{A, 12}}{V_{A, 2}}\left(\vec{Z}_{2}-\mu_{2}\right) \\
V_{A C} & =V_{A, 1}-\frac{C_{A, 12}^{2}}{V_{A, 2}} .
\end{aligned}
$$

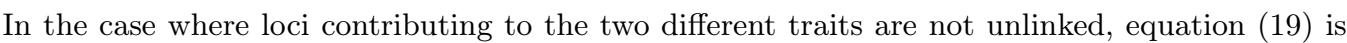
not an appropriate expression for the additive genetic covariance among traits, as it will depend also on the structure of linkage disequilibrium among sites. Because we ascertain SNPs independently for the two different traits, we expect that we will frequently have cases where two SNPs affecting two different traits within the same block are in linkage disequilibrium with one another, and therefore do not drift or respond to selection independently. To deal with this issue, we represent the genetic covariance among populations with a general form

$$
C_{A, 12}=\rho \sqrt{V_{A, 1} V_{A, 2}}
$$


where $\rho$ represents the genetic correlation between the two sets of polygenic scores. Further, we treat this genetic correlation parameter as an unknown, and also allow for one choice of genetic correlation parameter $\left(\rho_{1}\right)$ to describe the response of the mean, and a second correlation parameter to describe effects on the variance. The final two trait conditional model is then

$$
\begin{aligned}
\vec{Z}_{1} & \sim M V N\left(\xi, V_{A C} \mathbf{F}\right) \\
\xi & =\mu_{1}+\rho_{1}\left(\vec{Z}_{2}-\mu_{2}\right) \\
V_{A C} & =V_{A, 1}\left(1-\rho_{2}\right) .
\end{aligned}
$$

We calculate a conditional version of our test statistic:

$$
\frac{\left(Z_{1}-\xi\right)^{T} \mathbf{F}^{-1}\left(Z_{1}-\xi\right)}{V_{A C}}
$$

for a two dimensional grid of values for both $\rho_{1}$ and $\rho_{2}$ ranging from -1 to 1 and report the most conservative test. This procedure is overconservative, and therefore any trait which cannot be adequately explained explained as a response to some other trait under this framework is assume to have experienced an independent response to selection.

\section{S1.6.2 Alternate Ascertainment Tests}

As a second test, we constructed polygenic scores for each of HIP, WHR, IHC, and WC (which we denote with the prefix hsnp) using the subset of height SNPs for which an effect size estimate was available (about 1300 in each case), and then applied our test to these polygenic scores. Both hsnpHIP and hsnpWC show strong evidence of selection $\left(p=1.16 \times 10^{-14}\right.$ and $p=3.16 \times 10^{-6}$ respectively), while hsnpIHC and hsnpWHR each shows no convincing signal ( $p=0.07$ and $p=0.28$ respectively).

Combined with our results from the conditional model described above, this suggests that selection on height (or something tightly correlated to it) has plausibly impacted the genetic basis of HIP, WC, and probably IHC (though this alternate ascertainment test does not show evidence of, the conditional test does, and the moderate genetic correlation between them suggests it is likely). However, the conditional test cannot fully explain patterns observed for IHC and WHR given height, suggesting the action of independent selection. It is also conspicuous that we observe a stronger signal of selection for hsnpWC that for WC itself, and that the two are negatively correlated after accounting for population structure $\left(r=-0.24, p=9.5 \times 10^{-4}\right.$; though the presence of structure actually serves to mask the correlation; see Figure $\mathbf{S W}$ ). Given the moderate positive genetic correlation between height and WC within populations, this negative correlation is surprising, and suggests that WC has been impacted by selection independent of height. WHR seems the most plausible candidate of the phenotypes included in our study. 


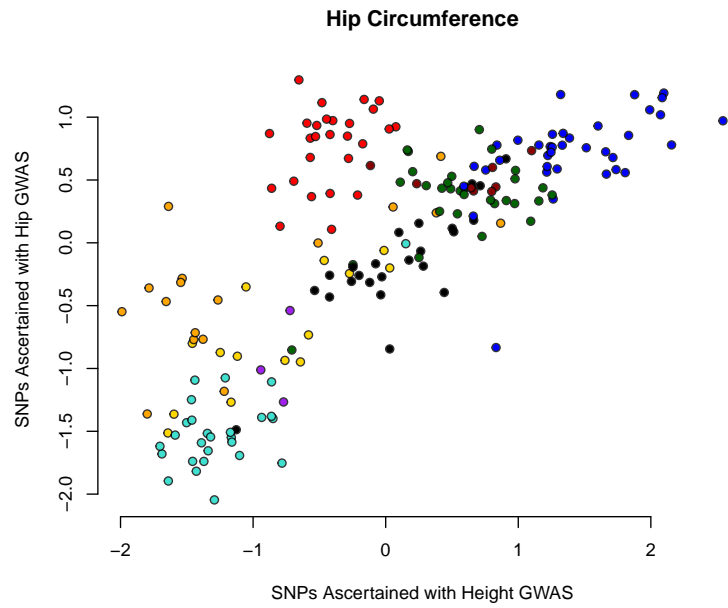

Infant Head Circumference

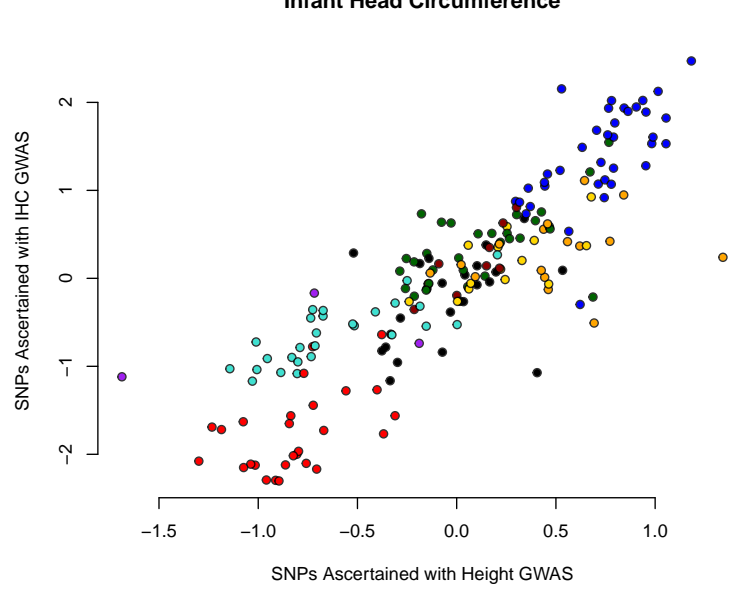

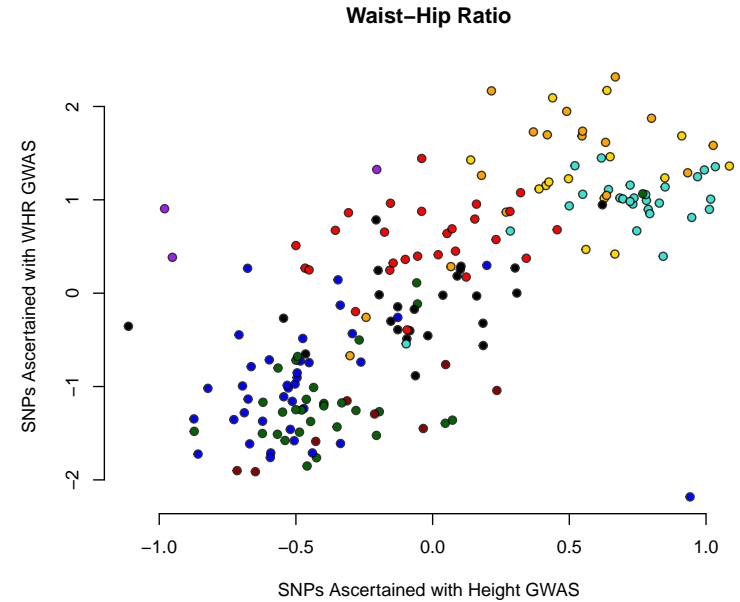

Waist Circumference

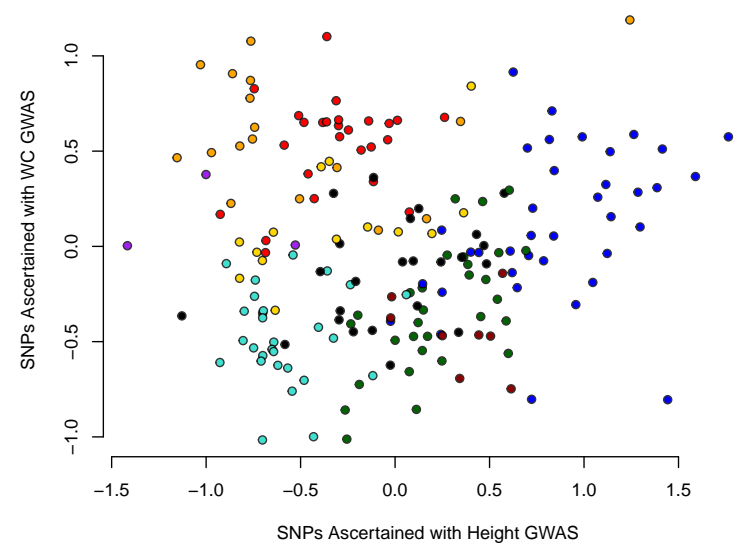

Figure S11: Polygenic scores for HIP, WHR, IHC, and WC plotted against scores computed from SNPs ascertained on the basis of association with height using the nominal effect size for the focal trait. 


\section{S1.7 Allometry}

Many phenotypes have allometric relationships, ${ }^{90,91}$ e.g. waist circumference does not increase linearly with the height across individuals within a population measured at the same growth stage(s) (termed individual or static allometry ${ }^{92}$ ). One natural concern therefore is that in ruling out that selection signal for (say) waist circumference can be completely explained by a correlated response to selection on height, we have not dealt with the allometric relationship among the phenotypes across populations. ${ }^{93}$ Our linear prediction of (say) waist-circumference conditional the observed polygenic scores for height in theory will not capture the non-linear relationship between the two phenotypes, and our claim of an independent signals in height and waist circumference might be suspect. The easiest way to see that this may not be too much of a concern is to note that while our results are significant the differences in polygenic score we see among populations all correspond to relatively small shifts in phenotype. Therefore, even though the phenotypes have an allometric relationship this will be approximately linear over the scale we are looking over and so well accounted for by our multivariate approach. To demonstrate this more thoroughly we convert our polygenic scores to a phenotype-scale, multiplying by the standard deviation of the phenotype and adding the mean phenotype, and plot two phenotypes on a log-log axes (e.g. height to waist circumference top panel in Figure (512), noting again that we do not view these as accurate phenotypic predictions). Confirming the idea that our deviations are small in phenotypic space, the log-log (base $e$ ) axes gives a very similar picture to the linear axes (top and bottom panel of Figure 512) suggesting that allometric scaling is not a concern.

We can however offer a more general response to the concerns about allometry, based on the fact that we construct our polygenic scores based only on the contribution of each locus to the additive variance (and no higher variance components). An observed allometric relationship between the underlying genetic phenotypes implies non-additive genetic covariance among the phenotypes (see e.g. Rice $\left.(1998)^{94}\right)$. To see this note that an allele with a fixed additive effect on height has a variable additive effect on waist circumference that depends on the distribution of allele frequencies at all of the height loci in the genome. For example, as WC has a positive allometric relationship with height, in a population with a high frequency of short height alleles the effect of our fixed height allele on waist circumference is smaller than when the population consists of many tall height alleles. Therefore, an allometric relationship between a pair of genetic phenotypes (among individuals or populations) implies that there is dominance and epistatic genetic covariance among the loci contributing to our traits (and some amount of epistatic variance in one or both traits). However, our polygenic scores are strictly based on the additive effect sizes, therefore they can not capture these higher order covariance components. As we are missing these higher order covariance terms our polygenic scores can fail to predict the phenotypes correctly due to allometry, but importantly also there can only be a linear relationship between our polygenic scores. Therefore our inferences of independent selection on 
the polygenic scores of multiple phenotypes cannot be confounded by allometry between phenotypes.

\section{S1.8 Height and the "thermoregulatory hypothesis".}

To study the "thermoregulatory hypothesis", Ruff $(1994)^{2}$ proposed a simple geometric model of body shape, where individuals are imagined as cylinders. The height of the cylinder is given by the individual's height $(h)$, and the cylinder's circumference $(C)$ by waist or hip circumference (note that Ruff modeled the diameter of the cylinder by bi-iliac breadth). Based on this we can calculate the surface area as $S=h C+4 \pi(C / 2 \pi)^{2}$; the volume as $V=2 \pi(C / 2 \pi)^{2} h$; and their ratio $V / S$. Based on these relationships we can compute the effect size of a SNP on surface, volume, and volume/surface ratio $\left(\alpha_{\mathrm{Surf}}, \alpha_{\mathrm{Vol}}, \alpha_{\mathrm{Vol} / \mathrm{Surf}}\right)$ based on the effect of a SNP on height and waist circumference $\left(\alpha_{\text {Height }}\right.$, $\left.\alpha_{\text {Waist }}\right)$, using a first order Taylor series approximation. ${ }^{95}$

We applied this procedure to all of our height SNPs, using the estimates of their effects on height and waist circumference, and in Figure 5 T3 we plot their effect on cylinder surface, volume, and volume/surface. Using the effect of height SNPs effect on hip circumference yields qualitatively similar results. Increasing height does indeed have a positive effect on $\mathrm{V} / \mathrm{S}$ ratio. However, as is to be expected from the weak direct dependence of a cylinder's $\mathrm{V} / \mathrm{S}$ ratio on height, this is almost entirely driven by the correlated effect of SNPs on (waist or hip) circumference. As we cannot explain our height signal as a correlated effect of selection on hip or waist circumference, it seems unlikely that selection on height is mediated only through selection on $\mathrm{V} / \mathrm{S}$ ratio.

\section{S1.9 Allen's Rule and Sitting Height Ratio}

To explore the effect of selection on leg and torso length we took our set of height SNPs and extracted their effect on sitting height ratio (SHR) from a SHR $\mathrm{GWAS}^{59}$ ). We found no net relationship between an allele's effect on height and it's effect on SHR (Spearman's $\rho=-0.010$, p-value $=0.66$, Figure S14A). This suggests that height SNPs act both on leg and head+torso length, as well some SNPs that affect each trait somewhat separately, see Chan et al. ${ }^{59}$ for more discussion. We compute a polygenic score from the effects of these height SNPs on SHR and found no signal of over-dispersion $\left(Q_{X}\right.$ p-value $\left.=0.19\right)$. This suggests that while selection has driven divergence in polygenic height scores, we do not have evidence that this selection has driven differences in the body-proportions of height.

To extend this observation we used the effect size of height SNPs on height and SHR ratio to estimate the unobserved effect of height SNPs on leg and torso+head height. We denoted the unknown effect sizes of the $\ell^{t h}$ SNP on leg and torso+head length by $\alpha_{L \ell}$ and $\alpha_{T \ell}$. The additive effect sizes of a SNPs on height and SHR are $\alpha_{H \ell}$ and $\alpha_{R \ell}$ respectively. While we will denote the 

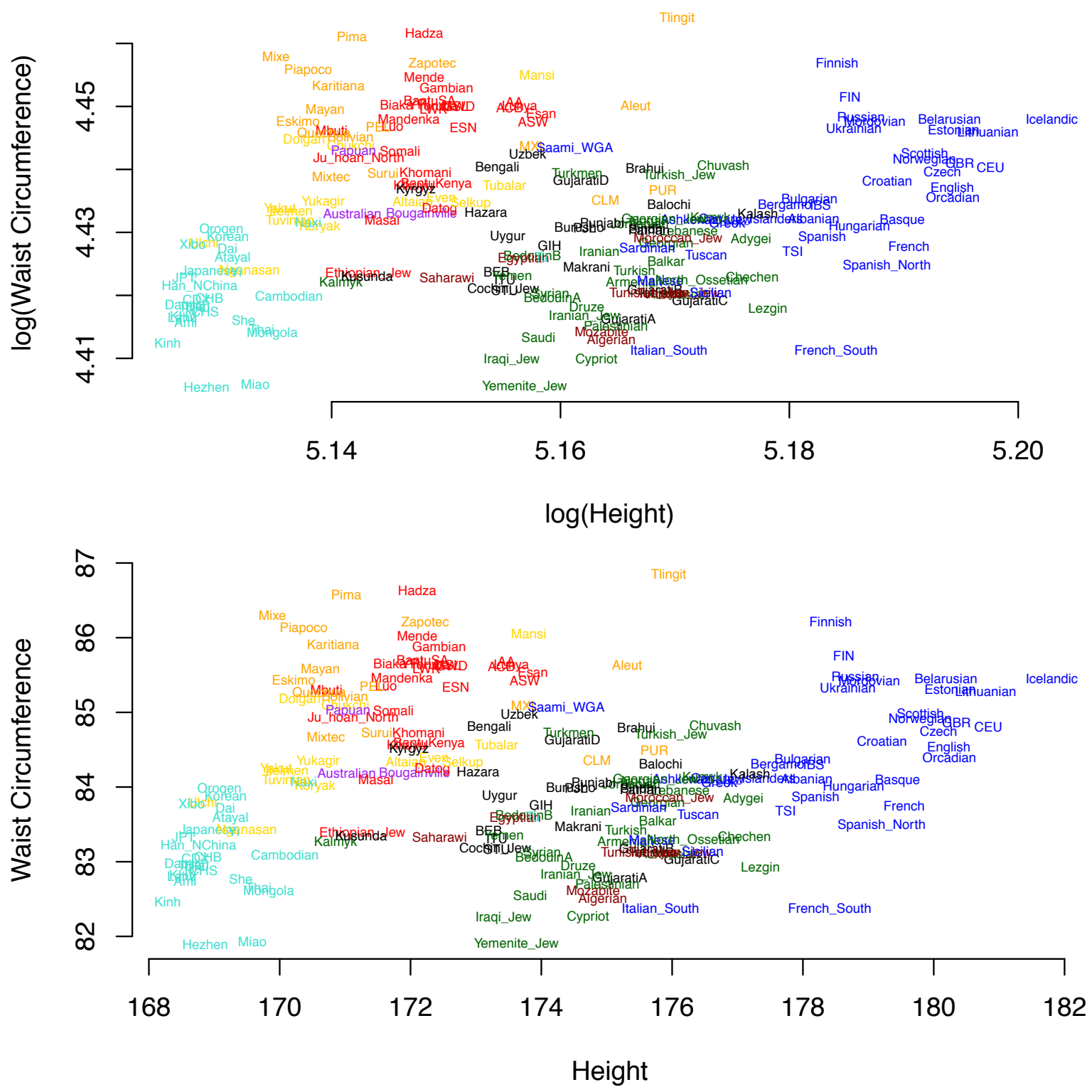

Figure S12: Relationship between polygenic scores, placed on phenotypic scale, for height and waist circumference on a log-log axis (top) and standard axes (bottom) 
bioRxiv preprint doi: https://doi org/101101/167551; this version posted April 18,2019 . The copyright holder for this preprint (which was not certified by peer review) is the author/funder, who has granted bioRxiv a license to display the preprint in perpetuity. It is made available under aCC-BY-NC 4.0 International license.
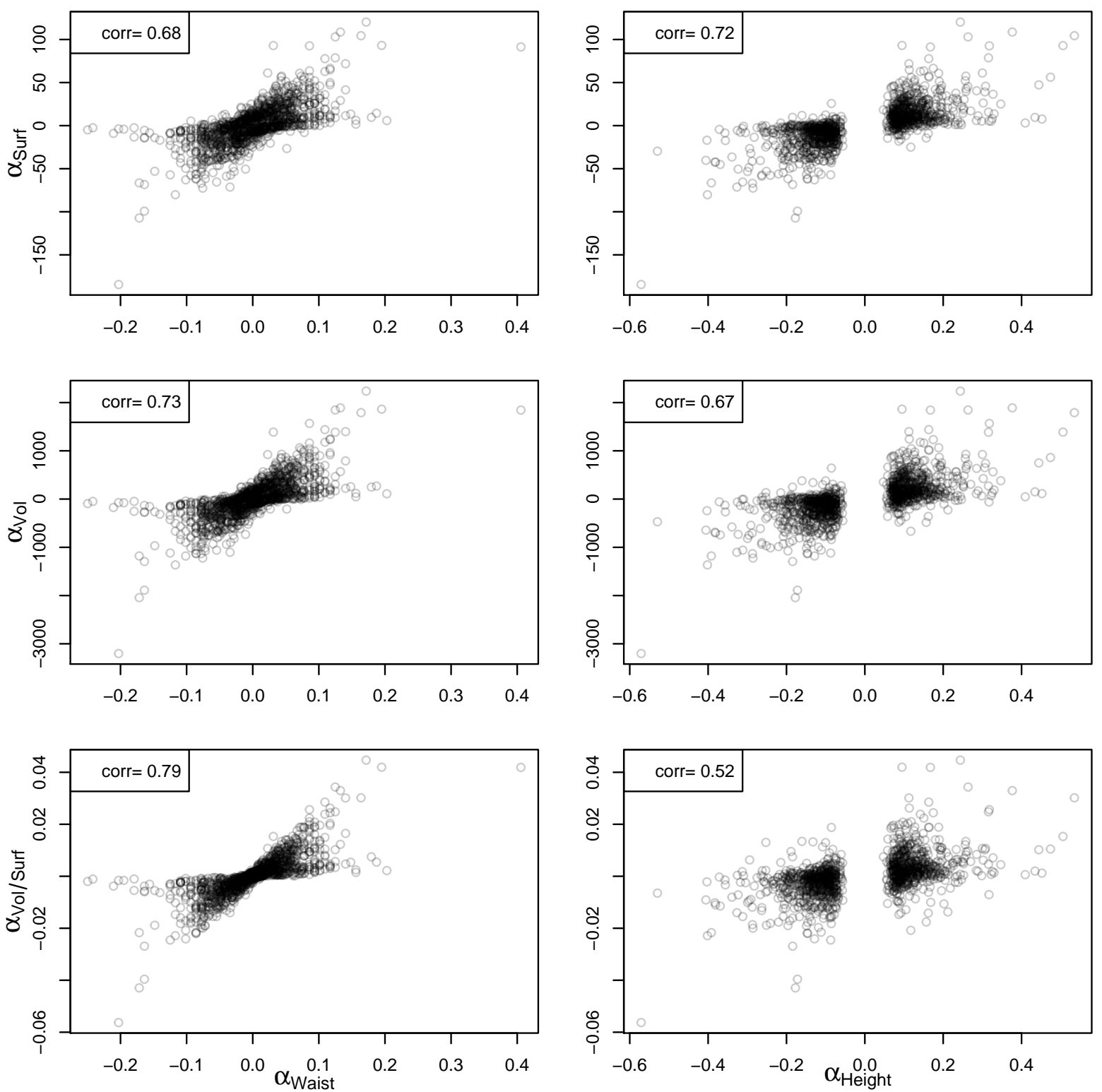

Figure S13: Relationship between the effect size of a SNP allele on height and waist circumference and the predicted effect of a SNP on surface area and volume. The pearson correlation coefficient between the two variables is shown in the top right corner of each plot. 
standard errors of these effect sizes by $\sigma_{H, \ell}$ and $\sigma_{L \ell}$. We model the additive height effect size as

$$
\alpha_{H, \ell} \sim N\left(\alpha_{L \ell}+\alpha_{T \ell}, \sigma_{H \ell}\right)
$$

and, by a first-order Taylor series approximation, the SHR ratio effect size is modeled as

$$
\alpha_{R \ell} \sim N\left(\alpha_{T \ell}-\mu_{R}\left(\alpha_{T \ell}+\alpha_{L \ell}\right), \sigma_{R \ell}\right)
$$

where $\mu_{R}$ is the population mean sitting height ratio $\left(\mu_{R}=0.52\right) .{ }^{59}$ We assume that the parameter pair $\left(\alpha_{L \ell}, \alpha_{T \ell}\right) \sim N(0, \Omega)$, over all our loci, where $\Omega$ is the $2 \times 2$ variance-covariance matrix between leg and torso+head effect sizes. We place hyper priors on the diagonal elements of $\Omega\left(\Omega_{i, i} \sim\right.$ Cauchy $\left.(0,1), \Omega_{i, i} \geq 0\right)$ and on the covariance $\left(\Omega_{1,2}=\rho \sqrt{\Omega_{1,1} \Omega_{2,2}}, \rho \sim U(-1,1)\right)$. We then estimate $\alpha_{L \ell}$ and $\alpha_{T \ell}$ over all loci. In Figure 514B-F we plot $\alpha_{L \ell}$ and $\alpha_{T \ell}$ against $\alpha_{H \ell}$ and $\alpha_{R \ell}$ and each other.

Using these estimates of effect sizes for leg and torso+head length we obtained $Q_{X}$ p-values of $4.0 \times 10^{-32}$ and $1.5 \times 10^{-31}$ respectively in our total sample. Thus both leg length and torso + head length show a strong signal of responding to selection on height. 

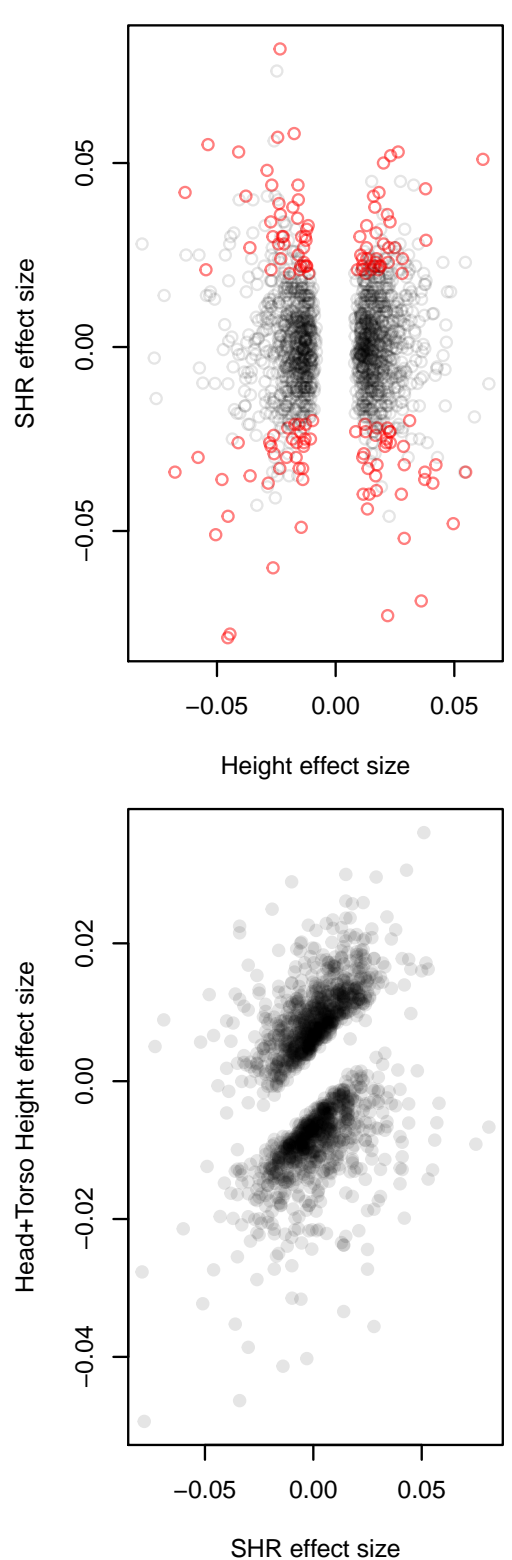
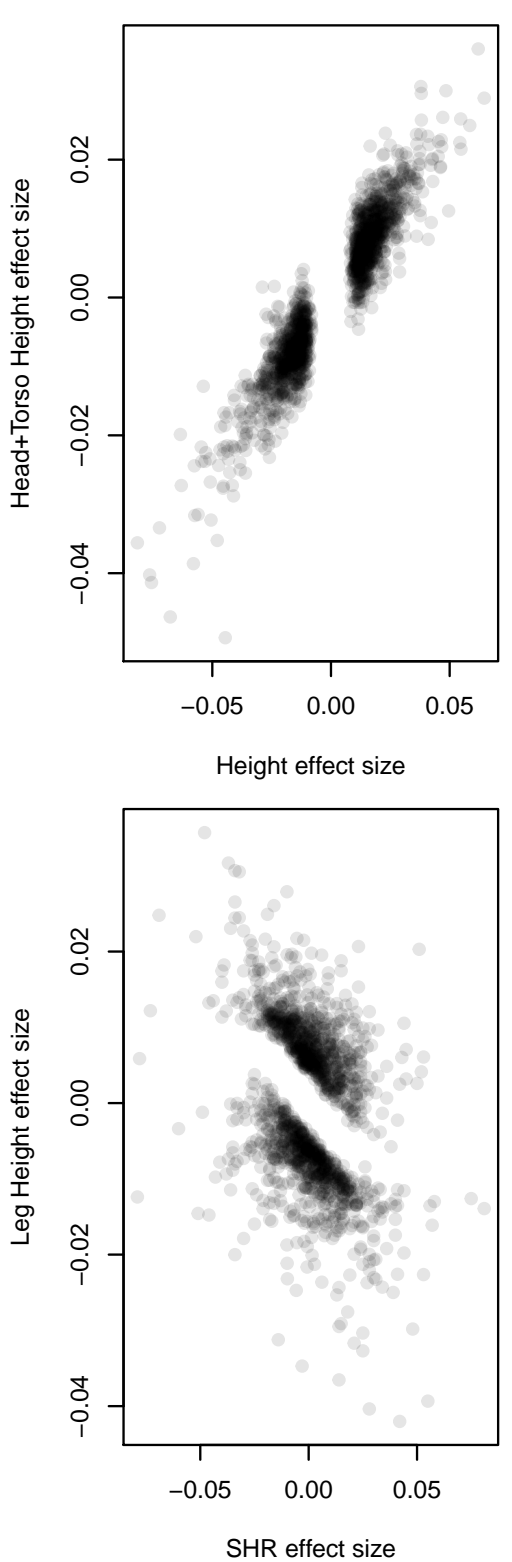
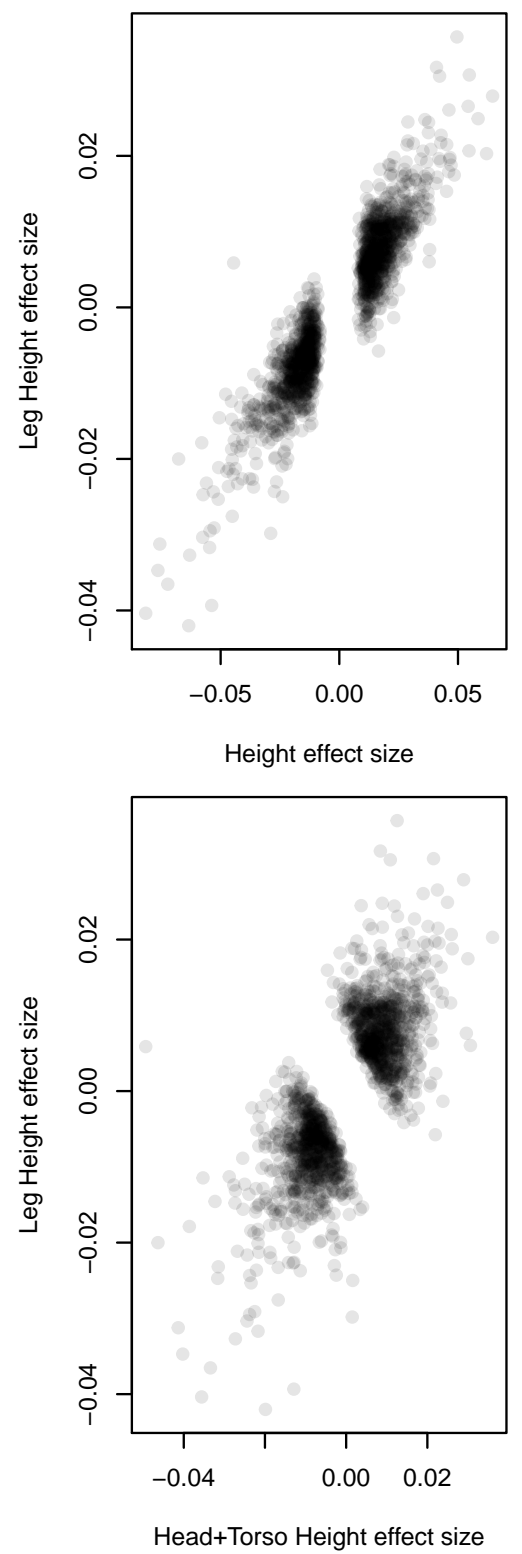

Figure S14: Plots of SNP effect sizes for height $\left(\alpha_{H \ell}\right)$ and SHR $\left(\alpha_{R \ell}\right)$ and the estimated effect sizes for leg $\left(\alpha_{L \ell}\right)$ and torso + head length $\left(\alpha_{T \ell}\right)$ plotted against each other over SNPs. See Section $\mathbf{S L} \mathbf{g}$ for more details. In the top left panel SNPs that have a significant effect on SHR $(p<0.05$ are colored orange.) 